\title{
Polymorphic System I
}

\author{
Cristian F. Sottile ${ }^{1} \quad$ Alejandro Díaz-Caro ${ }^{1,2}$ \\ Pablo E. Martínez López ${ }^{2}$ \\ ${ }^{1}$ Instituto de Investigación en Ciencias de la Computación (ICC). \\ CONICET-Universidad de Buenos Aires. Argentina. \\ ${ }^{2}$ Departamento de Ciencia y Tecnología. \\ Universidad Nacional de Quilmes. Argentina.
}

\begin{abstract}
System I is a simply-typed lambda calculus with pairs, extended with an equational theory obtained from considering the type isomorphisms as equalities. In this work we propose an extension of System I to polymorphic types, adding the corresponding isomorphisms. We provide non-standard proofs of subject reduction and strong normalisation, extending those of System I.
\end{abstract}

\section{Introduction}

Two types $A$ and $B$ are considered isomorphic (三) if there exist two functions $f$ of type $A \Rightarrow B$ and $g$ of type $B \Rightarrow A$ such that the composition $g \circ f$ is semantically equivalent to the identity in $A$ and the composition $f \circ g$ is semantically equivalent to the identity in $B$. Di Cosmo et al. [9] characterised the isomorphic types in different systems: simple types, simple types with pairs, polymorphism, etc. Using this characterisation, System I has been defined [12]. It is a simply-typed lambda calculus with pairs, where isomorphic types are considered equal. In this way, if $A$ and $B$ are isomorphic, every term of type $A$ can be used as a corresponding term of type $B$. For example, the currying isomorphism $(A \wedge B) \Rightarrow C \equiv A \Rightarrow B \Rightarrow C$ allows passing arguments one by one to a function expecting a pair. Normally, this would imply for a function $f:(A \wedge B) \Rightarrow C$ to be transformed through a term $r$ into rf : $A \Rightarrow B \Rightarrow C$. System I goes further, by considering that $f$ has both types $(A \wedge B) \Rightarrow C$ and $A \Rightarrow B \Rightarrow C$, and so, the transformation occurs implicitly without the need for the term $r$. To make this idea work, System I includes an equivalence between terms; for example: $r\langle s, t\rangle \rightleftarrows r s t$, since if $r$ expects a pair, it can also take each component at a time. Also, $\beta$-reduction has to be parametrized by the type: if the expected argument is a pair, then $r\langle s, t\rangle \beta$-reduces; otherwise, it does not $\beta$-reduce, but $r s t$ does. For example, $\left(\lambda x^{A \wedge B} . u\right)\langle r, s\rangle \beta$-reduces if $r$ has type $A$ and $s$ has type $B$. Instead, $\left(\lambda x^{A} . u\right)\langle r, s\rangle$ does not reduce directly, but since it is equivalent to $\left(\lambda x^{A} . u\right) r s$, which does reduce, then it also reduces, modulo this equivalence.

The idea of identifying some propositions has already been investigated, for example, in Martin-Löf's type theory [21], in the Calculus of Constructions [6], and in Deduction modulo theory [16, 17], where definitionally equivalent propositions, for instance $A \subseteq B, A \in \mathcal{P}(B)$, and $\forall x(x \in A \Rightarrow x \in B)$ can be identified. But definitional equality does not handle isomorphisms. For example, $A \wedge B$ and $B \wedge A$ are not identified in these logics. Besides definitional equality, identifying isomorphic types in type theory is also a goal of the univalence axiom [26]. From the programming perspective, isomorphisms capture the computational meaning correspondence between types. Taking currying again, for example, we have a function $f$ of type $A \wedge B \Rightarrow C$ that can be transformed, because there exists an isomorphism, into a function $f^{\prime}$ of type $A \Rightarrow B \Rightarrow C$. These two functions differ in how they can be combined with other terms, but they share a purpose: they both compute the same value of type $C$ given two arguments of types $A$ and $B$. In this sense, System I's proposal is to allow a programmer to focus on the meaning of programs, combining any term with the ones that are combinable with its isomorphic counterparts (e.g. $f x^{A} y^{B}$ and $f^{\prime}\left\langle x^{A}, y^{B}\right\rangle$ ), ignoring the rigid syntax of terms within the safe context provided by type isomorphisms. From the logic perspective, isomorphisms make proofs more natural. For instance, to prove $(A \wedge(A \Rightarrow B)) \Rightarrow B$ in natural deduction we need to introduce the conjunctive hypothesis $A \wedge(A \Rightarrow B)$, which has to be decomposed into $A$ and $A \Rightarrow B$, while using currying allows to transform 


$$
\begin{aligned}
A \wedge B & \equiv B \wedge A \\
A \wedge(B \wedge C) & \equiv(A \wedge B) \wedge C \\
A \Rightarrow(B \wedge C) & \equiv(A \Rightarrow B) \wedge(A \Rightarrow C) \\
(A \wedge B) \Rightarrow C & \equiv A \Rightarrow B \Rightarrow C \\
\text { If } X \notin F T V(A), \forall X .(A \Rightarrow B) & \equiv A \Rightarrow \forall X . B \\
\forall X .(A \wedge B) & \equiv \forall X . A \wedge \forall X . B
\end{aligned}
$$

Table 1: Isomorphisms considered in PSI

the goal to $A \Rightarrow(A \Rightarrow B) \Rightarrow B$ and to directly introduce the hypotheses $A$ and $A \Rightarrow B$, completely eliminating the need for the conjunctive hypotheses.

One of the pioneers on using isomorphisms in programming languages has been Rittri [24], who used the types, equated by isomorphisms, as search keys in program libraries.

An interpreter of a preliminary version of System I extended with a recursion operator has been implemented in Haskell [15]. Such a language have peculiar characteristics. For example, using the existing isomorphism between $A \Rightarrow(B \wedge C)$ and $(A \Rightarrow B) \wedge(A \Rightarrow C)$, we can project a function computing a pair of elements, and obtain, through evaluation, a simpler function computing only one of the elements of the pair, discarding the unused code that computes the output that is not of interest to us. That paper includes some non trivial examples.

In this work we propose an extension of System I to polymorphism, considering the corresponding isomorphisms.

Plan of the paper. The paper is organised as follows: Section 2 introduces the proposed system, and Section 3 gives examples to better clarify the constructions. Section 4 proves the Subject Reduction property and Section 5 the Strong Normalisation property, which are the main theorems in the paper. Finally, Section 6 discusses some design choices, as well as possible directions for future work.

\section{$2 \quad$ Intuitions and Definitions}

We define Polymorphic System I (PSI) as an extension of System I [12] to polymorphic types. The syntax of types coincides with that of System F [20, Chapter 11] with pairs:

$$
A:=\quad X|A \Rightarrow A| A \wedge A \mid \forall X . A
$$

where $X \in \mathcal{T}$ Var, a set of type variables.

The extension with respect to System $\mathrm{F}$ with pairs consists of adding a typing rule such that if $t$ has type $A$ and $A \equiv B$, then $t$ has also type $B$, which is valid for every pair of isomorphic types $A$ and $B$. This non-trivial addition induces a modification of the operational semantics of the calculus.

There are eight isomorphisms characterising all the valid isomorphisms of System F with pairs (cf. [9, Table 1.4]). From those eight, we consider the six given as a congruence in Table 1, where $F T V(A)$ is the set of free type variables defined as usual.

The two non-listed isomorphisms are the following:

$$
\begin{gathered}
\forall X . A \equiv \forall Y .[X:=Y] A \\
\forall X . \forall Y . A \equiv \forall Y . \forall X . A
\end{gathered}
$$

The isomorphism (7) is in fact an $\alpha$-equivalence, and we indeed consider terms and types modulo $\alpha$ equivalence. We simply do not make this isomorphism explicit in order to avoid confusion. The isomorphism (8), on the other hand, is not treated in this paper because PSI is presented in Church style (as System I), and so, being able to swap the arguments of a type abstraction would imply swapping the typing arguments with a cumbersome notation and little gain. We discuss this in Section 6.1. 
The added typing rule for isomorphic types induces certain equivalences between terms. In particular, the isomorphism (1) implies that the pairs $\langle r, s\rangle$ and $\langle s, r\rangle$ are indistinguishable, since both are typed as $A \wedge B$ and also as $B \wedge A$, independently of which term has type $A$ and which one type $B$. Therefore, we consider that those two pairs are equivalent. In the same way, as a consequence of isomorphism (2), $\langle r,\langle s, t\rangle\rangle$ is equivalent to $\langle\langle r, s\rangle, t\rangle$.

Such an equivalence between terms implies that the usual projection, which is defined with respect to the position (i.e. $\left.\pi_{i}\left(\left\langle r_{1}, r_{2}\right\rangle\right) \hookrightarrow r_{i}\right)$, is not well-defined in this system. Indeed, $\pi_{1}(\langle r, s\rangle)$ would reduce to $r$, but since $\langle r, s\rangle$ is equivalent to $\langle s, r\rangle$, it would also reduce to $s$. Therefore, PSI (as well as System I), defines the projection with respect to a type: if $\Gamma \vdash r: A$, then $\pi_{A}(\langle r, s\rangle) \hookrightarrow r$.

This rule turns PSI into a non-deterministic (and therefore non-confluent) system. Indeed, if both $r$ and $s$ have type $A$, then $\pi_{A}(\langle r, s\rangle)$ reduces non-deterministically to $r$ or to $s$. This non-determinism, however, can be argued not to be a major problem: if we think of PSI as a proof system, then the nondeterminism, as soon as we have type preservation, implies that the system identifies different proofs of isomorphic propositions (as a form of proof-irrelevance). On the other hand, if PSI is thought as a programming language, then the determinism can be recovered by the following encoding: if $r$ and $s$ have the same type, it suffices to encode the deterministic projection of $\langle r, s\rangle$ into $r$ as $\pi_{B \Rightarrow A}\left(\left\langle\lambda x^{B} . r, \lambda x^{C} . s\right\rangle\right) t$ where $B \not \equiv C$ and $t$ has type $B$. Hence, the non-determinism of System I (inherited in PSI) is considered a feature and not a flaw (cf. [12] for a longer discussion).

Thus, PSI (as well as System I) is one of the many non-deterministic calculi in the literature, e.g. [4, $5,7,8,22]$ and so our pair-construction operator can also be considered as the parallel composition operator of a non-deterministic calculus.

In non-deterministic calculi, the non-deterministic choice is such that if $r$ and $s$ are two $\lambda$-terms, the term $r \oplus s$ represents the computation that runs either $r$ or $s$ non-deterministically, that is such that $(r \oplus s) t$ reduces either to $r t$ or $s t$. On the other hand, the parallel composition operator $\|$ is such that the term $(r \| s) t$ reduces to $r t \| s t$ and continue running both $r t$ and $s t$ in parallel. In our case, given $r$ and $s$ of type $A \Rightarrow B$ and $t$ of type $A$, the term $\pi_{B}(\langle r, s\rangle t)$ is equivalent to $\pi_{B}(\langle r t, s t\rangle)$, which reduces to $r t$ or $s t$, while the term $\langle r t, s t\rangle$ itself would run both computations in parallel. Hence, our pair-constructor is equivalent to the parallel composition while the non-deterministic choice $\oplus$ is decomposed into the pair-constructor followed by its destructor.

In PSI and System I, the non-determinism comes from the interaction of two operators, $\langle$,$\rangle and \pi$. This is also related to the algebraic calculi $[1,2,3,27,11,14]$, some of which have been designed to express quantum algorithms. There is a clear link between our pair constructor and the projection $\pi$, with the superposition constructor + and the measurement $\pi$ on these algebraic calculi. In these cases, the pair $s+t$ is not interpreted as a non-deterministic choice, but as a superposition of two processes running $s$ and $t$, and the operator $\pi$ is the projection related to the measurement, which is the only non-deterministic operator. In such calculi, the distributivity rule $(r+s) t \rightleftarrows r t+s t$ is seen as the point-wise definition of the sum of two functions.

The syntax of terms is then similar to that of System $\mathrm{F}$ with pairs, but with the projections depending on types instead of position, as discussed:

$$
r \quad:=\quad x^{A}\left|\lambda x^{A} . r\right| r r|\langle r, r\rangle| \pi_{A}(r)|\Lambda X . r| r[A]
$$

where $x^{A} \in \mathcal{V}$ ar, a set of typed variables. We omit the type of variables when it is evident from the context. For example, we write $\lambda x^{A} \cdot x$ instead of $\lambda x^{A} \cdot x^{A}$.

The type system of PSI is standard, with only two modifications with respect to that of System F with pairs: the projection $\left(\wedge_{e}\right)$, and the added rule for isomorphisms $(\equiv)$. The full system is shown in Table 2. We write $\Gamma \vdash r: A$ to express that $r$ has type $A$ in context $\Gamma$. Notice, however, that since the system is given in Church-style (i.e. variables have their type written), the context is redundant [19, 23]. Hence, we may write " $r$ has type $A$ " with no ambiguity. From now on, except where indicated, we use the first upper-case letters of the Latin alphabet $(A, B, C, \ldots)$ for types, the last upper-case letters of the Latin alphabet $(W, X, Y, Z)$ for type variables, lower-case Latin letters $(r, s, t, \ldots)$ for terms, the last lower-case letters of the Latin alphabet $(x, y, z)$ for term variables, and upper-case Greek letters $(\Gamma, \Delta, \ldots)$ for contexts.

In the same way as isomorphisms (1) and (2) induce the commutativity and associativity of pairs, as well as a modification in the elimination of pairs (i.e. the projection), the isomorphism (3) induces that an abstraction of type $A \Rightarrow(B \wedge C)$ can be considered as a pair of abstractions of type $(A \Rightarrow$ 


$$
\begin{gathered}
\overline{\Gamma, x: A \vdash x: A}^{(\mathrm{ax})} \\
\frac{\Gamma \vdash r: A \quad A \equiv B}{\Gamma \vdash r: B}(\equiv) \\
\frac{\Gamma, x: A \vdash r: B}{\Gamma \vdash \lambda x^{A} \cdot r: A \Rightarrow B}\left(\Rightarrow_{i}\right) \\
\frac{\Gamma \vdash r: A \Rightarrow B \quad \Gamma \vdash s: A}{\Gamma \vdash r s: B}\left(\Rightarrow_{e}\right) \\
\frac{\Gamma \vdash r: A \quad \Gamma \vdash s: B}{\Gamma \vdash\langle r, s\rangle: A \wedge B}\left(\wedge_{i}\right) \\
\frac{\Gamma \vdash r: A \wedge B}{\Gamma \vdash \pi_{A}(r): A}\left(\wedge_{e}\right) \\
\frac{\Gamma \vdash r: A \quad X \notin F T V(\Gamma)}{\Gamma \vdash \Lambda X \cdot r: \forall X \cdot A}\left(\forall_{i}\right) \\
\frac{\Gamma \vdash r: \forall X . A}{\Gamma \vdash r[B]:[X:=B] A}\left(\forall_{e}\right)
\end{gathered}
$$

Table 2: Typing rules

$B) \wedge(A \Rightarrow C)$, and so it can be projected. Therefore, an abstraction returing a pair is identified with a pair of abstractions, and a pair applied distributes its argument- that is, $\lambda x^{A} .\langle r, s\rangle \rightleftarrows\left\langle\lambda x^{A} . r, \lambda x^{A} \cdot s\right\rangle$, and $\langle r, s\rangle t \rightleftarrows\langle r t, s t\rangle$, where $\rightleftarrows$ is a symmetric symbol (and $\rightleftarrows^{*}$ its transitive closure).

In addition, isomorphism (4) induces the following equivalence: $r\langle s, t\rangle \rightleftarrows r s t$. However, this equivalence produces an ambiguity with the $\beta$-reduction. For example, if $s$ has type $A$ and $t$ has type $B$, the term $\left(\lambda x^{A \wedge B} . r\right)\langle s, t\rangle$ can $\beta$-reduce to $[x:=\langle s, t\rangle] r$, but also, since this term is equivalent to $\left(\lambda x^{A \wedge B} . r\right) s t$, which $\beta$-reduces to $([x:=s] r) t$, reduction would not be stable by equivalence. To ensure the stability of reduction through equivalence, the $\beta$-reduction must be performed only when the type of the argument is the same as the type of the abstracted variable: if $\Gamma \vdash s: A$, then $\left(\lambda x^{A} . r\right) s \hookrightarrow[x:=s] r$.

The two added isomorphisms for polymorphism ((5) and (6)) also add several equivalences between terms. Two induced by (5), and four induced by (6).

Summarising, the operational semantics of PSI is given by the relation $\hookrightarrow$ modulo the symmetric relation $\rightleftarrows$. That is, we consider the relation

$$
\rightarrow \quad:=\rightleftarrows^{*} \circ \hookrightarrow \circ \rightleftarrows^{*}
$$

As usual, we write $\rightarrow^{*}$ the reflexive and transitive closure of $\rightarrow$. We also may write $\hookrightarrow^{n}$ to express $n$ steps in relation $\hookrightarrow$, and $\hookrightarrow_{R}$ to specify that the used rule is $R$. Both relations for PSI are given in Table 3.

\section{$3 \quad$ Examples}

In this Section we present some examples to discuss uses and necessity for the rules presented.

Example 3.1. We show the use of term equivalences to allow applications that are not possible to build in System F. For instance, the "apply" function

$$
\lambda f^{A \Rightarrow B} \cdot \lambda x^{A} \cdot f x
$$

can be applied to a pair, e.g. $\langle g, r\rangle$ with $\vdash g: A \Rightarrow B$ and $\vdash r: A$, because, due to isomorphism (4), the type derivation from Table 4 is valid. Then we have

$$
\left(\lambda f^{A \Rightarrow B} \cdot \lambda x^{A} \cdot f x\right)\langle g, r\rangle \rightleftarrows\left(\lambda f^{A \Rightarrow B} \cdot \lambda x^{A} \cdot f x\right) g r \hookrightarrow_{\beta_{\lambda}}^{2} g r
$$




$$
\begin{aligned}
& \langle r, s\rangle \rightleftarrows\langle s, r\rangle \\
& \langle r,\langle s, t\rangle\rangle \rightleftarrows\langle\langle r, s\rangle, t\rangle \\
& \lambda x^{A} \cdot\langle r, s\rangle \rightleftarrows\left\langle\lambda x^{A} . r, \lambda x^{A} . s\right\rangle \\
& \langle r, s\rangle t \rightleftarrows\langle r t, s t\rangle \\
& r\langle s, t\rangle \rightleftarrows r s t \\
& \text { If } X \notin F T V(A), \Lambda X . \lambda x^{A} . r \rightleftarrows \lambda x^{A} . \Lambda X . r \\
& \text { If } X \notin F T V(A),\left(\lambda x^{A} . r\right)[B] \rightleftarrows \lambda x^{A} . r[B] \\
& \Lambda X .\langle r, s\rangle \rightleftarrows\langle\Lambda X . r, \Lambda X . s\rangle \\
& \langle r, s\rangle[A] \rightleftarrows\langle r[A], s[A]\rangle \\
& \pi_{\forall X . A}(\Lambda X . r) \rightleftarrows \Lambda X . \pi_{A}(r) \\
& \text { If } r: \forall X .(B \wedge C),\left(\pi_{\forall X . B}(r)\right)[A] \rightleftarrows \pi_{[X:=A] B}(r[A]) \\
& \left(\operatorname{DIST}_{\lambda}\right) \\
& \text { (DISTapp) } \\
& \text { (CURRY) } \\
& \left(\text { P-Cомм }{ }_{\forall_{i} \rightarrow i}\right) \\
& \left(\mathrm{P}-\mathrm{COMM}_{\forall_{e} \rightrightarrows_{i}}\right) \\
& \left(\mathrm{P}-\mathrm{DIST}_{\forall_{i} \wedge_{i}}\right) \\
& \left(\text { P-DIST } \operatorname{Pe}_{\mathrm{e}} \wedge_{i}\right) \\
& \left(\text { P-DIST } \forall_{\forall_{i} \wedge}\right) \\
& \left(\text { P-DIST }_{\forall e} \wedge_{e}\right) \\
& \text { If } \Gamma \vdash s: A,\left(\lambda x^{A} . r\right) s \hookrightarrow[x:=s] r \\
& (\Lambda X . r)[A] \hookrightarrow[X:=A] r \\
& \text { If } \Gamma \vdash r: A, \pi_{A}(\langle r, s\rangle) \hookrightarrow r \\
& \frac{r \rightleftarrows s}{\lambda x^{A} . r \rightleftarrows \lambda x^{A} . s} \quad \frac{r \rightleftarrows s}{r t \rightleftarrows s t} \quad \frac{r \rightleftarrows s}{t r \rightleftarrows t s} \\
& \frac{r \rightleftarrows s}{\langle t, r\rangle \rightleftarrows\langle t, s\rangle} \quad \frac{r \rightleftarrows s}{\pi_{A}(r) \rightleftarrows \pi_{A}(s)} \quad \frac{r \rightleftarrows s}{\langle r, t\rangle \rightleftarrows\langle s, t\rangle} \\
& \frac{r \rightleftarrows s}{\Lambda X . r \rightleftarrows \Lambda X . s} \quad \frac{r \rightleftarrows s}{r[A] \rightleftarrows s[A]} \\
& \frac{r \hookrightarrow s}{\lambda x^{A} . r \hookrightarrow \lambda x^{A} . s} \quad \frac{r \hookrightarrow s}{r t \hookrightarrow s t} \quad \frac{r \hookrightarrow s}{t r \hookrightarrow t s} \\
& \frac{r \hookrightarrow s}{\langle t, r\rangle \hookrightarrow\langle t, s\rangle} \quad \frac{r \hookrightarrow s}{\pi_{A}(r) \hookrightarrow \pi_{A}(s)} \quad \frac{r \hookrightarrow s}{\langle r, t\rangle \hookrightarrow\langle s, t\rangle} \\
& \frac{r \hookrightarrow s}{\Lambda X . r \hookrightarrow \Lambda X . s} \quad \frac{r \hookrightarrow s}{r[A] \hookrightarrow s[A]}
\end{aligned}
$$

Table 3: Relations defining the operational semantics of PSI

Example 3.2. Continuing with the previous example, equivalent applications can be build in other ways. For instance, the term

$$
\left(\lambda f^{A \Rightarrow B} \cdot \lambda x^{A} \cdot f x\right) r g
$$

is well-typed using isomorphisms (1) and (4), and reduces to $g r$ :

$$
\begin{aligned}
\left(\lambda f^{A \Rightarrow B} \cdot \lambda x^{A} \cdot f x\right) r g & \rightleftarrows\left(\lambda f^{A \Rightarrow B} \cdot \lambda x^{A} \cdot f x\right)\langle r, g\rangle \\
& \rightleftarrows\left(\lambda f^{A \Rightarrow B} \cdot \lambda x^{A} \cdot f x\right)\langle g, r\rangle \\
& \rightarrow^{*} g r
\end{aligned}
$$

Example 3.3. Concluding with the previous example, the uncurried "apply" function

$$
\lambda z^{(A \Rightarrow B) \wedge A} \cdot \pi_{A \Rightarrow B}(z) \pi_{A}(z)
$$

can be applied to $\vdash g: A \Rightarrow B$ and $\vdash r: A$ as if it was curried:

$$
\begin{aligned}
& \left(\lambda z^{(A \Rightarrow B) \wedge A} \cdot \pi_{A \Rightarrow B}(z) \pi_{A}(z)\right) g r \\
& \rightleftarrows\left(\lambda z^{(A \Rightarrow B) \wedge A} \cdot \pi_{A \Rightarrow B}(z) \pi_{A}(z)\right)\langle g, r\rangle
\end{aligned}
$$




$$
\frac{\frac{\vdash \lambda f^{A \Rightarrow B} \cdot \lambda x^{A} \cdot f x:(A \Rightarrow B) \Rightarrow A \Rightarrow B}{\vdash \lambda f^{A \Rightarrow B} \cdot \lambda x^{A} \cdot f x:((A \Rightarrow B) \wedge A) \Rightarrow B}(\equiv) \quad \frac{\vdash g: A \Rightarrow B \quad \vdash r: A}{\vdash\langle g, r\rangle:(A \Rightarrow B) \wedge A}\left(\wedge_{i}\right)}{\vdash\left(\lambda f^{A \Rightarrow B} \cdot \lambda x^{A} \cdot f x\right)\langle g, r\rangle: B}\left(\Rightarrow_{e}\right)
$$

Table 4: Type derivation of example 3.1.



Table 5: Type derivation of example 3.5 .

$$
\begin{aligned}
& \hookrightarrow_{\beta_{\lambda}} \pi_{A \Rightarrow B}(\langle g, r\rangle) \pi_{A}(\langle g, r\rangle) \\
& \hookrightarrow_{\pi}^{2} g r
\end{aligned}
$$

In the three previous examples, the $\beta$-reduction cannot occur before the equivalences because of the typing condition in rule $\left(\beta_{\lambda}\right)$.

Example 3.4. Another use of interest is the one mentioned in Section 2: a function returning a pair can be projected even while not being applied, computing another function. Consider the term

$$
\pi_{A \Rightarrow B}\left(\lambda x^{A} \cdot\langle r, s\rangle\right)
$$

where $x: A \vdash r: B$ and $x: A \vdash s: C$. This term is typable using isomorphism (3), since $A \Rightarrow(B \wedge C) \equiv$ $(A \Rightarrow B) \wedge(A \Rightarrow C)$. The reduction goes as follows:

$$
\begin{aligned}
\pi_{A \Rightarrow B}\left(\lambda x^{A} \cdot\langle r, s\rangle\right) & \rightleftarrows \pi_{A \Rightarrow B}\left(\left\langle\lambda x^{A} \cdot r, \lambda x^{A} \cdot s\right\rangle\right) \\
& \hookrightarrow_{\pi} \lambda x^{A} \cdot r
\end{aligned}
$$



$$
\left(\Lambda X . \lambda x^{A} \cdot \lambda f^{A \Rightarrow X} \cdot f x\right) r
$$

is well-typed assuming $\vdash r: A$ and $X \notin F T V(A)$, as shown in Table 5, and we have

$$
\begin{aligned}
\left(\Lambda X . \lambda x^{A} \cdot \lambda f^{A \Rightarrow X} \cdot f x\right) r & \rightleftarrows\left(\lambda x^{A} \cdot\left(\Lambda X . \lambda f^{A \Rightarrow X} \cdot f x\right)\right) r \\
& \hookrightarrow \beta_{\lambda}\left(\Lambda X . \lambda f^{A \Rightarrow X} \cdot f r\right)
\end{aligned}
$$

Example 3.6. Rule (

$$
\left(\lambda x^{\forall X .(X \Rightarrow X)} \cdot x\right)[A] \Lambda X \cdot \lambda x^{X} \cdot x
$$

Let $B=\forall X .(X \Rightarrow X)$. Since $B \Rightarrow B \equiv \forall Y .(B \Rightarrow(Y \Rightarrow Y))$ (renaming the variable for readability), then

$$
\vdash\left(\lambda x^{B} \cdot x\right)[A] \Lambda X \cdot \lambda x^{X} \cdot x: A \Rightarrow A
$$

The reduction goes as follows:

$$
\begin{aligned}
& \left(\lambda x^{\forall X .(X \Rightarrow X)} \cdot x\right)[A] \Lambda X \cdot \lambda x^{X} \cdot x \\
& \rightleftarrows\left(\lambda x^{\forall X .(X \Rightarrow X)} \cdot x[A]\right) \Lambda X \cdot \lambda x^{X} \cdot x \\
& \hookrightarrow \beta_{\lambda}\left(\Lambda X \cdot \lambda x^{X} \cdot x\right)[A] \hookrightarrow \beta_{\Lambda} \lambda x^{A} \cdot x
\end{aligned}
$$

Example 3.7. Rules ( $\left.\mathrm{P}-\mathrm{DIST}_{\forall_{i} \wedge_{i}}\right)$ and ( P-DIST$_{\forall_{i} \wedge_{e}}$ ) are both consequences of the same isomorphism: (6). Consider the term

$$
\pi_{\forall X .(X \Rightarrow X)}\left(\Lambda X \cdot\left\langle\lambda x^{X} \cdot x, r\right\rangle\right)
$$


where $\vdash r: A$. Since $\forall X .((X \Rightarrow X) \wedge A) \equiv(\forall X .(X \Rightarrow X)) \wedge \forall X . A$, we can derive

$$
\vdash \pi_{\forall X .(X \Rightarrow X)}\left(\Lambda X .\left\langle\lambda x^{X} \cdot x, r\right\rangle\right): \forall X .(X \Rightarrow X)
$$

A possible reduction is:

$$
\begin{aligned}
& \pi_{\forall X .(X \Rightarrow X)}\left(\Lambda X .\left\langle\lambda x^{X} . x, r\right\rangle\right) \\
& \rightleftarrows \pi_{\forall X .(X \Rightarrow X)}\left(\left\langle\Lambda X . \lambda x^{X} . x, \Lambda X . r\right\rangle\right) \\
& \hookrightarrow_{\pi} \Lambda X . \lambda x^{X} . x
\end{aligned}
$$

Example 3.8. Rule (P-DIST ${ }_{\forall_{e} \wedge_{i}}$ ) is also a consequence of isomorphism (6). Consider

$$
\left\langle\Lambda X . \lambda x^{X} \cdot \lambda y^{A} \cdot r, \Lambda X \cdot \lambda x^{X} \cdot \lambda z^{B} \cdot s\right\rangle[C]
$$

where $\vdash r: D$ and $\vdash s: E$. It has type $(C \Rightarrow A \Rightarrow D) \wedge(C \Rightarrow B \Rightarrow E)$, and reduces as follows:

$$
\begin{aligned}
& \left\langle\Lambda X \cdot \lambda x^{X} \cdot \lambda y^{A} \cdot r, \Lambda X \cdot \lambda x^{X} \cdot \lambda z^{B} \cdot s\right\rangle[C] \\
& \rightleftarrows\left\langle\left(\lambda x^{X} \cdot \lambda y^{A} \cdot r\right)[C],\left(\lambda x^{X} \cdot \lambda z^{B} \cdot s\right)[C]\right\rangle \\
& \hookrightarrow_{\beta_{\Lambda}}\left\langle\lambda x^{C} \cdot \lambda y^{A} \cdot r, \lambda x^{C} \cdot \lambda z^{B} \cdot s\right\rangle
\end{aligned}
$$

Example 3.9. Rule (P-DIST $\left.{ }_{\forall_{e}} \wedge_{e}\right)$, too, is a consequence of isomorphism (6). Consider the term

$$
\left(\pi_{\forall X .(X \Rightarrow X)}\left(\Lambda X .\left\langle\lambda x^{X} \cdot x, r\right\rangle\right)\right)[A]
$$

with type $A \Rightarrow A$, which reduces as follows:

$$
\begin{aligned}
& \left(\pi_{\forall X .(X \Rightarrow X)}\left(\Lambda X .\left\langle\lambda x^{X} . x, r\right\rangle\right)\right)[A] \\
& \rightleftarrows \pi_{A \Rightarrow A}\left(\left(\Lambda X .\left\langle\lambda x^{X} . x, r\right\rangle\right)[A]\right) \\
& \hookrightarrow_{\beta_{\Lambda}} \pi_{A \Rightarrow A}\left(\left\langle\lambda x^{A} \cdot x,[X:=A] r\right\rangle\right) \\
& \hookrightarrow_{\pi} \lambda x^{A} . x
\end{aligned}
$$

\section{Subject reduction}

In this section we prove the preservation of typing through reduction. First we need to characterise the equivalences between types, for example, if $\forall X . A \equiv B \wedge C$, then $B \equiv \forall X . B^{\prime}$ and $C \equiv \forall X . C^{\prime}$, with $A \equiv B^{\prime} \wedge C^{\prime}$ (Lemma 4.9). Due to the amount of isomorphisms, this kind of lemmas are not trivial. To prove these relations, we first define the multiset of prime factors of a type (Definition 4.1). That is, the multiset of types that are not equivalent to a conjunction, such that the conjunction of all its elements is equivalent to a certain type. This technique has already been used in System I [12], however, it has been used with simple types with only one basic type $\tau$. In PSI, instead, we have an infinite amount of variables acting as basic types, hence the proof becomes more complex.

We write $\vec{X}$ for $X_{1}, \ldots, X_{n}$ and $\forall \vec{X} . A$ for $\forall X_{1} \ldots \forall X_{n} . A$, for some $n$ (where, in the second case, if $n=0, \forall \vec{X} \cdot A=A)$. In addition, we write $\left[A_{1}, \ldots, A_{n}\right]$ or $\left[A_{i}\right]_{i=1}^{n}$ for the multiset containing the elements $A_{1}$ to $A_{n}$.

Definition 4.1 (Prime factors).

$$
\begin{aligned}
P F(X) & =[X] \\
P F(A \Rightarrow B)=\left[\forall \vec{X}_{i} \cdot\left(\left(A \wedge B_{i}\right) \Rightarrow Y_{i}\right)\right]_{i=1}^{n} & \\
& \text { where } P F(B)=\left[\forall \vec{X}_{i} \cdot\left(B_{i} \Rightarrow Y_{i}\right)\right]_{i=1}^{n} \\
P F(A \wedge B)=P F(A) \uplus P F(B) & \\
P F(\forall X . A)=\left[\forall X . \forall \vec{Y}_{i} \cdot\left(A_{i} \Rightarrow Z_{i}\right)\right]_{i=1}^{n} & \text { where } P F(A)=\left[\forall \vec{Y}_{i} \cdot\left(A_{i} \Rightarrow Z_{i}\right)\right]_{i=1}^{n}
\end{aligned}
$$


Lemma 4.2 and Corollary 4.3 state the correctness of Definition 4.1 . We write $\bigwedge\left(\left[A_{i}\right]_{i=1}^{n}\right)$ for $\bigwedge_{i=1}^{n} A_{i}$.

Lemma 4.2. For all $A$, there exist $\vec{X}, n, B_{1}, \ldots, B_{n}, Y_{1}, \ldots, Y_{n}$ such that $P F(A)=\left[\forall \vec{X}_{i} \cdot\left(B_{i} \Rightarrow Y_{i}\right)\right]_{i=1}^{n}$.

Proof. Straightforward induction on the structure of $A$.

Corollary 4.3. For all $A, A \equiv \bigwedge(P F(A))$.

Proof. By induction on the structure of A.

- Let $A=X$. Then $P F(X)=[X]$, and $\bigwedge([X])=X$.

- Let $A=B \Rightarrow C$. By Lemma 4.2, $P F(C)=\left[\forall \vec{X}_{i} \cdot\left(C_{i} \Rightarrow Y_{i}\right)\right]_{i=1}^{n}$. Hence, by definition, $\operatorname{PF}(A)=$ $\left[\forall \vec{X}_{i} \cdot\left(B \wedge C_{i} \Rightarrow Y_{i}\right)\right]_{i=1}^{n}$. By the induction hypothesis, $C \equiv \bigwedge(P F(C))=\bigwedge_{i=1}^{n} \forall \vec{X}_{i} \cdot\left(C_{i} \Rightarrow Y_{i}\right)$. Therefore,

$$
\begin{aligned}
A=B \Rightarrow C & \equiv B \Rightarrow \bigwedge_{i=1}^{n} \forall \vec{X}_{i} \cdot\left(C_{i} \Rightarrow Y_{i}\right) \\
& \equiv \bigwedge_{i=1}^{n} \forall \vec{X}_{i} \cdot\left(\left(B \wedge C_{i}\right) \Rightarrow Y_{i}\right) \\
& =\bigwedge\left(\left[\forall \vec{X}_{i} \cdot\left(B \wedge C_{i} \Rightarrow Y_{i}\right)\right]_{i=1}^{n}\right)=\bigwedge(P F(A))
\end{aligned}
$$

- Let $A=B \wedge C$. By the induction hypothesis, $B \equiv \bigwedge(P F(B))$ and $C \equiv \bigwedge(P F(C))$. Hence,

$$
\begin{aligned}
A & =B \wedge C \equiv \bigwedge(P F(B)) \wedge \bigwedge(P F(C)) \\
& \equiv \bigwedge(P F(B) \uplus P F(C))=\bigwedge(P F(A))
\end{aligned}
$$

- Let $A=\forall X . B$. By Lemma 4.2, $P F(B)=\left[\forall \vec{Y}_{i} \cdot\left(B_{i} \Rightarrow Z_{i}\right)\right]_{i=1}^{n}$. Hence, by definition, $P F(A)=$ $\left[\forall X . \forall \vec{Y}_{i} \cdot\left(B_{i} \Rightarrow Z_{i}\right)\right]_{i=1}^{n}$. By the induction hypothesis, $B \equiv \bigwedge(P F(B))=\bigwedge_{i=1}^{n} \forall \vec{Y}_{i} \cdot\left(B_{i} \Rightarrow Z_{i}\right)$. Therefore,

$$
\begin{aligned}
A & =\forall X \cdot B \equiv \forall X \cdot \bigwedge_{i=1}^{n} \forall \vec{Y}_{i} \cdot\left(B_{i} \Rightarrow Z_{i}\right) \\
& \equiv \bigwedge_{i=1}^{n} \forall X \cdot \forall \vec{Y}_{i} \cdot\left(B_{i} \Rightarrow Z_{i}\right) \\
& =\bigwedge\left(\left[\forall X \cdot \forall \vec{Y} \cdot\left(B_{i} \Rightarrow Z\right)\right]_{i=1}^{n}\right)=\bigwedge(P F(A))
\end{aligned}
$$

Lemma 4.5 states the stability of prime factors through equivalence and Lemma 4.6 states a kind of reciprocal result.

Definition 4.4. $\left[A_{1}, \ldots, A_{n}\right] \sim\left[B_{1}, \ldots, B_{m}\right]$ if $n=m$ and $A_{i} \equiv B_{p(i)}$, for $i=1, \ldots, n$ and $p$ a permutation on $\{1, \ldots n\}$.

Lemma 4.5. For all $A, B$ such that $A \equiv B$, we have $\operatorname{PF}(A) \sim P F(B)$.

Proof. First we check that $P F(A \wedge B) \sim P F(B \wedge A)$ and similar for the other five isomorphisms. Then we prove by structural induction that if $A$ and $B$ are equivalent in one step, then $P F(\mathrm{~A}) \sim P F(\mathrm{~B})$. We conclude by an induction on the length of the derivation of the equivalence $A \equiv B$.

Lemma 4.6. For all $R, S$ multisets such that $R \sim S$, we have $\bigwedge(R) \equiv \bigwedge(S)$.

Lemma 4.7. For all $\vec{X}, \vec{Z}, A, B, Y, W$ such that $\forall \vec{X} .(A \Rightarrow Y) \equiv \forall \vec{Z} .(B \Rightarrow W)$, we have $\vec{X}=\vec{Z}, A \equiv B$, and $Y=W$. 
Proof. By simple inspection of the isomorphisms.

Lemma 4.8. For all $A, B, C_{1}, C_{2}$ such that $A \Rightarrow B \equiv C_{1} \wedge C_{2}$, there exist $B_{1}, B_{2}$ such that $C_{1} \equiv A \Rightarrow B_{1}$, $C_{2} \equiv A \Rightarrow B_{2}$ and $B \equiv B_{1} \wedge B_{2}$.

Proof. By Lemma 4.5, $\operatorname{PF}(A \Rightarrow B) \sim \operatorname{PF}\left(C_{1} \wedge C_{2}\right)=\operatorname{PF}\left(C_{1}\right) \uplus P F\left(C_{2}\right)$.

By Lemma 4.2 , let $P F(B)=\left[\forall \vec{X}_{i} \cdot\left(D_{i} \Rightarrow Z_{i}\right)\right]_{i=1}^{n}, P F\left(C_{1}\right)=\left[\forall \vec{Y}_{j} \cdot\left(E_{j} \Rightarrow Z_{j}^{\prime}\right)\right]_{j=1}^{k}$, and $P F\left(C_{2}\right)=$ $\left[\forall \vec{Y}_{j} \cdot\left(E_{j} \Rightarrow Z_{j}^{\prime}\right)\right]_{j=k+1}^{m}$. Hence, $\left[\forall \vec{X}_{i} \cdot\left(\left(A \wedge D_{i}\right) \Rightarrow Z_{i}\right)\right]_{i=1}^{n} \sim\left[\forall \vec{Y}_{j} \cdot\left(E_{j} \Rightarrow Z_{j}^{\prime}\right)\right]_{j=1}^{m}$. So, by definition of $\sim$, $n=m$ and for $i=1, \ldots, n$ and a permutation $p$, we have $\forall \vec{X}_{i} \cdot\left(\left(A \wedge D_{i}\right) \Rightarrow Z_{i}\right) \equiv \forall \vec{Y}_{p(i)} \cdot\left(E_{p(i)} \Rightarrow Z_{p(i)}^{\prime}\right)$. Hence, by Lemma 4.7 , we have $\vec{X}_{i}=\vec{Y}_{p(i)}, A \wedge D_{i} \equiv E_{p(i)}$, and $Z_{i}=Z_{p(i)}^{\prime}$.

Thus, there exists $I$ such that $I \cup \bar{I}=\{1, \ldots, n\}$, such that

$$
\begin{aligned}
& P F\left(C_{1}\right)=\left[\forall \vec{Y}_{p(i)} \cdot\left(E_{p(i)} \Rightarrow Z_{p(i)}^{\prime}\right)\right]_{i \in I} \\
& P F\left(C_{2}\right)=\left[\forall \vec{Y}_{p(i)} \cdot\left(E_{p(i)} \Rightarrow Z_{p(i)}^{\prime}\right)\right]_{i \in \bar{I}}
\end{aligned}
$$

Therefore, by Corollary 4.3,

$$
C_{1} \equiv \bigwedge_{i \in I} \forall \vec{Y}_{p(i)} \cdot\left(E_{p(i)} \Rightarrow Z_{p_{i}}^{\prime}\right) \equiv \bigwedge_{i \in I} \forall \vec{X}_{i} \cdot\left(\left(A \wedge D_{i}\right) \Rightarrow Z_{i}\right)
$$

and

$$
C \equiv \bigwedge_{i \in \bar{I}} \forall \vec{X}_{i} \cdot\left(\left(A \wedge D_{i}\right) \Rightarrow Z_{i}\right)
$$

Let $B_{1}=\bigwedge_{i \in I} \forall \vec{X}_{i} \cdot\left(D_{i} \Rightarrow Z_{i}\right)$ and $B_{2}=\bigwedge_{i \in \bar{I}} \forall \vec{X}_{i} \cdot\left(D_{i} \Rightarrow Z_{i}\right)$. So, $C_{1} \equiv A \Rightarrow B_{1}$ and $C_{2} \equiv A \Rightarrow B_{2}$. In addition, also by Corollary 4.3 , we have $B \equiv \bigwedge_{i=1}^{n} \forall \vec{X}_{i} \cdot\left(D_{i} \Rightarrow Z_{i}\right) \equiv B_{1} \wedge B_{2}$.

The proofs of the following two lemmas are similar to the proof of Lemma 4.8. Full details are given in the technical appendix published at [25].

Lemma 4.9. For all $X, A, B, C$ such that $\forall X . A \equiv B \wedge C$, there exist $B^{\prime}, C^{\prime}$ such that $B \equiv \forall X . B^{\prime}$, $C \equiv \forall X . C^{\prime}$ and $A \equiv B^{\prime} \wedge C^{\prime}$.

Lemma 4.10. For all $X, A, B, C$ such that $\forall X . A \equiv B \Rightarrow C$, there exists $C^{\prime}$ such that $C \equiv \forall X . C^{\prime}$ and $A \equiv B \Rightarrow C^{\prime}$.

Since the calculus is presented in Church-style, excluding rule (三), PSI is syntax directed. Therefore, the generation lemma (Lemma 4.12) is straightforward, and we have the following unicity lemma (whose proof is given in the technical appendix published at [25]):

Lemma 4.11 (Unicity modulo). For all $\Gamma, r, A, B$ such that $\Gamma \vdash r: A$ and $\Gamma \vdash r: B$, we have $A \equiv B$.

Lemma 4.12 (Generation). For all $\Gamma, x, r, s, X, A, B$ :

1. If $\Gamma \vdash x: A$ and $\Gamma \vdash x: B$, then $A \equiv B$.

2. If $\Gamma \vdash \lambda x^{A} . r: B$, then there exists $C$ such that $\Gamma, x: A \vdash r: C$ and $B \equiv A \Rightarrow C$.

3. If $\Gamma \vdash r s: A$, then there exists $C$ such that $\Gamma \vdash r: C \Rightarrow A$ and $\Gamma \vdash s: C$.

4. If $\Gamma \vdash\langle r, s\rangle: A$, then there exist $C, D$ such that $A \equiv C \wedge D, \Gamma \vdash r: C$ and $\Gamma \vdash s: D$.

5. If $\Gamma \vdash \pi_{A}(r): B$, then $A \equiv B$ and there exists $C$ such that $\Gamma \vdash r: B \wedge C$.

6. If $\Gamma \vdash \Lambda X . r: A$, then there exists $C$ such that $A \equiv \forall X . C, \Gamma \vdash r: C$ and $X \notin F T V(\Gamma)$.

7. If $\Gamma \vdash r[A]: B$, then there exists $C$ such that $[X:=A] C \equiv B$ and $\Gamma \vdash r: \forall X . C$.

The detailed proofs of Lemma 4.13 (Substitution) and Theorem 4.14 (Subject Reduction) are given in the technical appendix at [25].

Lemma 4.13 (Substitution). 
1. For all $\Gamma, x, r, s, A, B$ such that $\Gamma, x: B \vdash r: A$ and $\Gamma \vdash s: B$, we have $\Gamma \vdash[x:=s] r: A$.

2. For all $\Gamma, r, X, A, B$ such that $\Gamma \vdash r: A$, we have $[X:=B] \Gamma \vdash[X:=B] r:[X:=B] A$.

Theorem 4.14 (Subject reduction). For all $\Gamma, r, s, A$ such that $\Gamma \vdash r: A$ and $r \hookrightarrow s$ or $r \rightleftarrows s$, we have $\Gamma \vdash s: A$.

\section{Strong Normalisation}

In this section we prove the strong normalisation of the relation $\rightarrow$, that is, every reduction sequence fired from a typed term eventually terminates. The set of typed strongly normalising terms with respect to reduction $\rightarrow$ is written SN. The size of the longest reduction issued from $t$ is written $|t|$.

We extend to polymorphism the proof of System I [12]. To prove that every term is in SN, we associate, as usual, a set $\llbracket A \rrbracket$ of strongly normalising terms to each type $A$. A term $\vdash r: A$ is said to be reducible when $r \in \llbracket A \rrbracket$. We then prove an adequacy theorem stating that every well typed term is reducible.

The set $\llbracket A_{1} \Rightarrow A_{2} \Rightarrow \cdots \Rightarrow A_{n} \Rightarrow X \rrbracket$ can be defined either as the set of terms $r$ such that for all $s \in \llbracket A_{1} \rrbracket, r s \in \llbracket A_{2} \Rightarrow \cdots \Rightarrow A_{n} \Rightarrow X \rrbracket$ or, equivalently, as the set of terms $r$ such that for all $s_{i} \in \llbracket A_{i} \rrbracket$, $r s_{1} \ldots s_{n} \in \llbracket X \rrbracket=\mathrm{SN}$. To prove that a term of the form $\lambda x^{A}$.t is reducible, we need to use the so-called CR3 property [20], in the first case, and the property that a term whose all one-step reducts are in SN is in SN, in the second. In PSI, an introduction can be equivalent to an elimination e.g. $\langle r t, s t\rangle \rightleftarrows\langle r, s\rangle t$, hence, we cannot define a notion of neutral term and have an equivalent to the CR3 property. Therefore, we use the second definition, and since reduction depends on types, the set $\llbracket A \rrbracket$ is defined as a set of typed terms.

Before proving the normalisation of PSI, we reformulate the proof of strong normalisation of System $\mathrm{F}$ along these lines.

\subsection{Normalisation of System F}

Definition 5.1 (Elimination context). Consider an extension of the language where we introduce an extra symbol $\left(D D^{A}\right.$, called hole of type $A$. We define the set of elimination contexts with a hole $\left(D^{A}\right.$ as the smallest set such that:

- $\left(D^{A}\right.$ is an elimination context of type $A$,

- if $K_{A}^{B \Rightarrow C}$ is an elimination context of type $B \Rightarrow C$ with a hole of type $A$ and $r \in \mathrm{SN}$ is a term of type $B$, then $K_{A}^{B \Rightarrow C} r$ is an elimination context of type $C$ with a hole of type $A$,

- and if $K_{A}^{\forall X . B}$ is an elimination context of type $\forall X . B$ with a hole of type $A$, then $K_{A}^{\forall X . B}[C]$ is an elimination context of type $[X:=C] B$ with a hole of type $A$.

We write $K_{A}^{B}\left(|r|\right.$ for $\left[0 D^{A}:=r\right]\left(K_{A}^{B}\right)$, where ()$^{A}$ is the hole of $K_{A}^{B}$. In particular, $r$ may be an elimination context.

Notice that the shape of every context $K_{A}^{B}$ is $\left(D^{A} \alpha_{1} \ldots \alpha_{n}\right.$, where each $\alpha_{i}$ is either a term or a type argument.

Example 5.2. Let

$$
\begin{aligned}
K_{X}^{X} & =\left(D^{X}\right. \\
K_{X \Rightarrow X}^{\prime X} & =K_{X}^{X}\left(0 D^{X \Rightarrow X} x D\right. \\
K_{\forall X . X \Rightarrow X}^{\prime \prime X} & \left.=K_{X \Rightarrow X}^{\prime X}(00)^{\forall X . X \Rightarrow X}[X]\right) \\
& =K_{X}^{X}\left(0 D^{\forall X . X \Rightarrow X}[X] x\right) D
\end{aligned}
$$

Then $K_{\forall X . X \Rightarrow X}^{\prime \prime X}\left(\Lambda X \cdot \lambda y^{X} . y\right)=\left(\Lambda X . \lambda y^{X} \cdot y\right)[X] x$. 
Definition 5.3 (Terms occurring in an elimination context). Let $K_{A}^{B}$ be an elimination context. The multiset of terms occurring in $K_{A}^{B}$ is defined as

$$
\begin{aligned}
\mathcal{T}\left(\left(0 D^{A}\right)\right. & =\emptyset \\
\mathcal{T}\left(K_{A}^{B \Rightarrow C} r\right) & =\mathcal{T}\left(K_{A}^{B \Rightarrow C}\right) \uplus\{r\} \\
\mathcal{T}\left(K_{A}^{\forall X . B}[C]\right) & =\mathcal{T}\left(K_{A}^{\forall X . B}\right)
\end{aligned}
$$

We write $\left|K_{A}^{B}\right|$ for $\sum_{i=1}^{n}\left|r_{i}\right|$ where $\left[r_{1}, \ldots, r_{n}\right]=\mathcal{T}\left(K_{A}^{B}\right)$.

Definition 5.4 (Reducibility). The set of reducible terms of type $A$ (notation $\llbracket A \rrbracket$ ) is defined as the set of terms $r$ of type $A$ such that for any elimination context $K_{A}^{X}$ where all the terms in $\mathcal{T}\left(K_{A}^{X}\right)$ are in SN, we have $K_{A}^{X}(r) \in \mathrm{SN}$.

Lemma 5.5. For all $A, \llbracket A \rrbracket \subseteq \mathrm{SN}$.

Proof. For all $A$, there exists an elimination context $K_{A}^{X}$, since variables are in $\mathrm{SN}$ and they can have any type. Hence, given that if $r \in \llbracket A \rrbracket$ then $K_{A}^{X}(r) \in \mathrm{SN}$, we have $r \in \mathrm{SN}$.

Lemma 5.6 (Adequacy of variables). For all $A$ and $x^{A}$, we have $x^{A} \in \llbracket A \rrbracket$.

Proof. Let $K_{A}^{X}=\left(D^{A} \alpha_{1} \ldots \alpha_{n}\right.$, where for all $i$ such that $\alpha_{i}$ is a term, we have $\alpha_{i} \in \mathrm{SN}$, then for all $x$, $K_{A}^{X}(x) \in \mathrm{SN}$.

Lemma 5.7 (Adequacy of application). For all $r, s, A, B$ such that $r \in \llbracket A \Rightarrow B \rrbracket$ and $s \in \llbracket A \rrbracket$, we have $r s \in \llbracket B \rrbracket$.

Proof. We need to prove that for every elimination context $K_{B}^{X}$, we have $K_{B}^{X}(r s) \in \mathrm{SN}$. Since $s \in \llbracket A \rrbracket$, $\left.K_{A \Rightarrow B}^{\prime X}=K_{B}^{X}(0){ }^{A \Rightarrow B} s\right\rangle \in \mathrm{SN}$, and $r \in \llbracket A \Rightarrow B \rrbracket$, we have $K_{A \Rightarrow B}^{\prime X}(r)=K_{B}^{X}(r s) \in \mathrm{SN}$.

Lemma 5.8 (Adequacy of abstraction). For all $t, r, x, A, B$ such that $t \in \llbracket A \rrbracket$ and $[x:=t] r \in \llbracket B \rrbracket$, we have $\lambda x^{A} . r \in \llbracket A \Rightarrow B \rrbracket$.

Proof. We need to prove that for every elimination context $K_{A \Rightarrow B}^{X}$, we have $K_{A \Rightarrow B}^{X}\left(\lambda x^{A} \cdot r\right) \in \mathrm{SN}$, that is that all its one step reducts are in SN. By Lemma 5.6, $x \in \llbracket A \rrbracket$, so $r \in \llbracket B \rrbracket \subseteq$ SN. We conclude with an induction on $|r|+\left|K_{A \Rightarrow B}^{X}\right|$.

Lemma 5.9 (Adequacy of type application). For all $r, X, A, B$ such that $r \in \llbracket \forall X$. $A \rrbracket$, we have $r[B] \in$ $\llbracket[X:=B] A \rrbracket$.

Proof. We need to prove that for every elimination context $K_{[X:=B] A}^{Y}$ we have $K_{[X:=B] A}^{Y}(r[B] D \in \mathrm{SN}$. Let $\left.K_{\forall X . A}^{\prime Y}=K_{[X:=B] A}^{Y}(0)^{\forall X . A}[B]\right) \in \mathrm{SN}$, and since $r \in \llbracket \forall X . A \rrbracket$, we have $K_{\forall X . A}^{\prime Y}(r)=K_{[X:=B] A}^{Y}(r[B]) \in$ SN.

Lemma 5.10 (Adequacy of type abstraction). For all $r, X, A, B$ such that $[X:=B] r \in \llbracket[X:=B] A \rrbracket$, we have $\Lambda X . r \in \llbracket \forall X . A \rrbracket$.

Proof. We need to prove that for every elimination context $K_{\forall X . A}^{Y}$, we have $K_{\forall X . A}^{Y}(\Lambda X . r) \in \mathrm{SN}$, that is that all its one step reducts are in SN. Since $[X:=B] r \in \llbracket A \rrbracket \subseteq \mathrm{SN}$ and every term in $\mathcal{T}\left(K_{\forall X . A}^{Y}\right)$ is in SN, then all its one step reducts are in SN.

Definition 5.11 (Adequate substitution). A substitution $\theta$ is adequate for a context $\Gamma$ (notation $\theta \vDash \Gamma$ ) if for all $x: A \in \Gamma$, we have $\theta(x) \in \llbracket A \rrbracket$.

Theorem 5.12 (Adequacy). For all $\Gamma, r, A$, and substitution $\theta$ such that $\Gamma \vdash r: A$ and $\theta \vDash \Gamma$, we have $\theta r \in \llbracket A \rrbracket$.

Proof. By induction on $r$, using Lemmas 5.6 to 5.10 .

Theorem 5.13 (Strong normalisation). For all $\Gamma, r, A$ such that $\Gamma \vdash r: A$, we have $r \in \mathrm{SN}$.

Proof. By Lemma 5.6, the idendity substitution is adequate. Thus, by Theorem 5.12 and Lemma 5.5, $r \in \llbracket A \rrbracket \subseteq \mathrm{SN}$. 


\subsection{Measure on terms}

The size of a term is not invariant through the equivalence $\rightleftarrows$. for example, counting the number of lambda abstractions in a term, we see that $\lambda x^{A} .\langle r, s\rangle$ is different than $\left\langle\lambda x^{A} . r, \lambda x^{A} . s\right\rangle$. Hence we introduce a measure $M(\cdot)$ on terms.

Definition 5.14 (Measure on terms).

$$
\begin{aligned}
P(x) & =0 \\
P\left(\lambda x^{A} . r\right) & =P(r) \\
P(r s) & =P(r) \\
P(\langle r, s\rangle) & =1+P(r)+P(s) \\
P\left(\pi_{A}(r)\right) & =P(r) \\
P(\Lambda X . r) & =P(r) \\
P(r[A]) & =P(r) \\
M(x) & =1 \\
M\left(\lambda x^{A} . r\right) & =1+M(r)+P(r) \\
M(r s) & =M(r)+M(s)+P(r) M(s) \\
M(\langle r, s\rangle) & =M(r)+M(s) \\
M\left(\pi_{A}(r)\right) & =1+M(r)+P(r) \\
M(\Lambda X . r) & =1+M(r)+P(r) \\
M(r[A]) & =1+M(r)+P(r)
\end{aligned}
$$

Lemma 5.15. For all $r, s$ such that $r \rightleftarrows s$, we have $P(r)=P(s)$.

Proof. We check the case of each rule of Table 3, and then conclude by structural induction to handle the contextual closure.

- (сомм): $P(\langle r, s\rangle)=1+P(r)+P(s)$

$$
=P(\langle s, r\rangle)
$$

- (Asso): $P(\langle\langle r, s\rangle, t\rangle)=2+P(r)+P(s)+P(t)$

$$
=P(\langle r,\langle s, t\rangle\rangle)
$$

- $\left(\operatorname{DIST}_{\lambda}\right): P\left(\lambda x^{A} \cdot\langle r, s\rangle\right)=1+P(r)+P(s)$

$$
=P\left(\left\langle\lambda x^{A} . r, \lambda x^{A} . s\right\rangle\right)
$$

- $\left(\right.$ DIST $\left._{\text {app }}\right): P(\langle r, s\rangle t)=1+P(r)+P(s)$

$$
=P(\langle r t, s t\rangle)
$$

- (curRy): $P((r s) t)=P(r)$

$$
=P(r\langle s, t\rangle)
$$

- $\left(\mathrm{P}-\mathrm{COMM}_{\forall_{i} \Rightarrow_{i}}\right): P\left(\Lambda X \cdot \lambda x^{A} \cdot r\right)=P(r)$

$$
=P\left(\lambda x^{A} \cdot \Lambda X . r\right)
$$

- $\left(\mathrm{P}-\mathrm{COMM}_{\forall_{e} \Rightarrow_{i}}\right): P\left(\left(\lambda x^{A} . r\right)[B]\right)=P(r)$

$$
=P\left(\lambda x^{A} \cdot r[B]\right)
$$

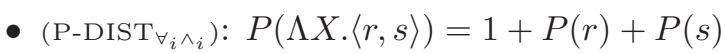

$$
=P(\langle\Lambda X . r, \Lambda X . s\rangle)
$$




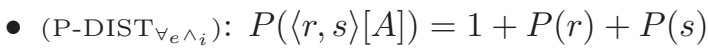

$$
=P(\langle r[A], s[A]\rangle)
$$

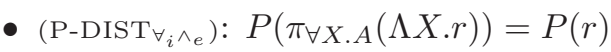

$$
=P\left(\Lambda X . \pi_{A}(r)\right)
$$

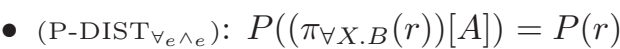

$$
=P\left(\pi_{[X:=A] B}(r[A])\right)
$$

Lemma 5.16. For all $r, s$ such that $r \rightleftarrows s$, we have $M(r)=M(s)$.

Proof. We check the case of each rule of Table 3, and then conclude by structural induction to handle the contextual closure.

- (сомм): $M(\langle r, s\rangle)$

$$
\begin{aligned}
& =M(r)+M(s) \\
& =M(\langle s, r\rangle)
\end{aligned}
$$

- (Asso): $M(\langle\langle r, s\rangle, t\rangle)$

$$
\begin{aligned}
& =M(r)+M(s)+M(t) \\
& =M(\langle r,\langle s, t\rangle\rangle)
\end{aligned}
$$

- (DIST $\lambda): M\left(\lambda x^{A} \cdot\langle r, s\rangle\right)$

$$
\begin{aligned}
& =2+M(r)+M(s)+P(r)+P(s) \\
& =M\left(\left\langle\lambda x^{A} . r, \lambda x^{A} . s\right\rangle\right)
\end{aligned}
$$

- (DISTapp): $M(\langle r, s\rangle t)$

$$
\begin{aligned}
= & M(r)+M(s)+2 M(t) \\
& +P(r) M(t)+P(s) M(t) \\
= & M(\langle r t, s t\rangle)
\end{aligned}
$$

- (curry): $M((r s) t)$

$$
\begin{aligned}
= & M(r)+M(s)+P(r) M(s) \\
& +M(t)+P(r) M(t) \\
= & M(r\langle s, t\rangle)
\end{aligned}
$$

- $\left(\right.$ Р-сомм $\left._{i} \Rightarrow_{i}\right): M\left(\Lambda X \cdot \lambda x^{A} \cdot r\right)$

$$
\begin{aligned}
& =2+M(r)+2 P(r) \\
& =M\left(\lambda x^{A} . \Lambda X . r\right)
\end{aligned}
$$

- $\left({\mathrm{P}-\text { сомм }_{\ell} \Rightarrow_{i}}_{i}\right): M\left(\left(\lambda x^{A} \cdot r\right)[B]\right)$

$$
\begin{aligned}
& =2+M(r)+2 P(r) \\
& =M\left(\lambda x^{A} . r[B]\right)
\end{aligned}
$$

- $\left(\mathrm{P}-\mathrm{DIST}_{\forall_{i} \wedge_{i}}\right): M(\Lambda X .\langle r, s\rangle)$

$$
\begin{aligned}
& =1+M(r)+M(s) \\
& =M(\langle\Lambda X . r, \Lambda X . s\rangle)
\end{aligned}
$$

- $\left({\mathrm{P}-\operatorname{DIST}_{\forall} \wedge_{i}}_{i}\right): M(\langle r, s\rangle[A])$

$$
\begin{aligned}
& =2+M(r)+P(r)+M(s)+P(s) \\
& =M(\langle r[A], s[A]\rangle)
\end{aligned}
$$


- $\left({\mathrm{P}-\mathrm{DIST}_{\forall_{i}} \wedge_{e}}\right): M\left(\pi_{\forall X . A}(\Lambda X . r)\right)$

$=2+M(r)+2 P(r)$

$=M\left(\Lambda X . \pi_{A}(r)\right)$

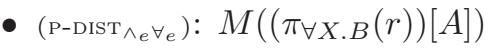

$=2+M(r)+2 P(r)$

$=M\left(\pi_{[X:=A] B}(r[A])\right)$

Lemma 5.17. For all $r, s, X, A$,

$$
\begin{aligned}
M\left(\lambda x^{A} . r\right) & >M(r) & M(\langle r, s\rangle) & >M(s) \\
M(r s) & >M(r) & M\left(\pi_{A}(r)\right) & >M(r) \\
M(r s) & >M(s) & M(\Lambda X . r) & >M(r) \\
M(\langle r, s\rangle) & >M(r) & M(r[A]) & >M(r)
\end{aligned}
$$

Proof. For all $t, M(t) \geq 1$. We conclude by case inspection.

\subsection{Reduction of a product}

When typed lambda-calculus is extended with pairs, proving that if $r_{1} \in \mathrm{SN}$ and $r_{2} \in \mathrm{SN}$ then $\left\langle r_{1}, r_{2}\right\rangle \in$ SN is easy. However, in System I and PSI this property (Lemma 5.20) is harder to prove, as it requires a characterisation of the terms equivalent to the product $\left\langle r_{1}, r_{2}\right\rangle$ (Lemma 5.18) and of all the reducts of this term (Lemma 5.19).

Lemma 5.18. For all $r, s, t$ such that $\langle r, s\rangle \rightleftarrows^{*} t$, we have either

1. $t=\langle u, v\rangle$ where either

(a) $u \rightleftarrows^{*}\left\langle t_{11}, t_{21}\right\rangle$ and $v \rightleftarrows^{*}\left\langle t_{12}, t_{22}\right\rangle$ with $r \rightleftarrows^{*}\left\langle t_{11}, t_{12}\right\rangle$ and $s \rightleftarrows^{*}\left\langle t_{21}, t_{22}\right\rangle$, or

(b) $v \rightleftarrows^{*}\langle w, s\rangle$ with $r \rightleftarrows^{*}\langle u, w\rangle$, or any of the three symmetric cases, or

(c) $r \rightleftarrows^{*} u$ and $s \rightleftarrows^{*} v$, or the symmetric case.

2. $t=\lambda x^{A}$. $a$ and $a \rightleftarrows^{*}\left\langle a_{1}, a_{2}\right\rangle$ with $r \rightleftarrows^{*} \lambda x^{A} . a_{1}$ and $s \rightleftarrows^{*} \lambda x^{A} . a_{2}$.

3. $t=a v$ and $a \rightleftarrows^{*}\left\langle a_{1}, a_{2}\right\rangle$, with $r \rightleftarrows^{*} a_{1} v$ and $s \rightleftarrows^{*} a_{2} v$.

4. $t=\Lambda X$. a and $a \rightleftarrows^{*}\left\langle a_{1}, a_{2}\right\rangle$ with $r \rightleftarrows^{*} \Lambda X . a_{1}$ and $s \rightleftarrows^{*} \Lambda X . a_{2}$.

5. $t=a[A]$ and $a \rightleftarrows^{*}\left\langle a_{1}, a_{2}\right\rangle$, with $r \rightleftarrows^{*} a_{1}[A]$ and $s \rightleftarrows^{*} a_{2}[A]$.

Proof. By a double induction, first on $M(t)$ and then on the length of the relation $\rightleftarrows^{*}$. Full details are given in the technical appendix at [25].

Lemma 5.19. For all $r_{1}, r_{2}, s, t$ such that $\left\langle r_{1}, r_{2}\right\rangle \rightleftarrows^{*} s \hookrightarrow t$, there exists $u_{1}, u_{2}$ such that $t \rightleftarrows^{*}\left\langle u_{1}, u_{2}\right\rangle$ and either

1. $r_{1} \rightarrow u_{1}$ and $r_{2} \rightarrow u_{2}$,

2. $r_{1} \rightarrow u_{1}$ and $r_{2} \rightleftarrows^{*} u_{2}$, or

3. $r_{1} \rightleftarrows^{*} u_{1}$ and $r_{2} \rightarrow u_{2}$.

Proof. By induction on $M\left(\left\langle r_{1}, r_{2}\right\rangle\right)$. Full details are given in the technical appendix at [25].

Lemma 5.20. For all $r_{1}, r_{2}$ such that $r_{1} \in \mathrm{SN}$ and $r_{2} \in \mathrm{SN}$, we have $\left\langle r_{1}, r_{2}\right\rangle \in \mathrm{SN}$.

Proof. By Lemma 5.19, from a reduction sequence starting from $\left\langle r_{1}, r_{2}\right\rangle$ we can extract one starting from $r_{1}$, from $r_{2}$ or from both. Hence, this reduction sequence is finite. 


\subsection{Reducibility}

Definition 5.21 (Elimination context). Consider an extension of the language where we introduce an extra symbol $\left(D^{A}\right.$, called hole of type $A$. We define the set of elimination contexts with a hole $\left(D^{A}\right.$ as the smallest set such that:

- $\left(D^{A}\right.$ is an elimination context of type $A$,

- if $K_{A}^{B \Rightarrow C}$ is an elimination context of type $B \Rightarrow C$ with a hole of type $A$ and $r \in \mathrm{SN}$ is a term of type $B$, then $K_{A}^{B \Rightarrow C} r$ is an elimination context of type $C$ with a hole of type $A$,

- if $K_{A}^{B \wedge C}$ is an elimination context of type $B \wedge C$ with a hole of type $A$, then $\pi_{B}\left(K_{A}^{B \wedge C}\right)$ is an elimination context of type $B$ with a hole of type $A$.

- and if $K_{A}^{\forall X . B}$ is an elimination context of type $\forall X . B$ with a hole of type $A$, then $K_{A}^{\forall X . B}[C]$ is an elimination context of type $[X:=C] B$ with a hole of type $A$.

We write $K_{A}^{B}(r)$ for $\left[O D^{A}:=r\right]\left(K_{A}^{B}\right)$, where $O D^{A}$ is the hole of $K_{A}^{B}$. In particular, $r$ may be an elimination context.

Example 5.22. Let

$$
\begin{aligned}
K_{X}^{X} & =()^{X}, \\
K_{X \Rightarrow(X \wedge X)}^{\prime X} & \left.=K_{X}^{X}\left(1 \pi_{X}(0)^{X \Rightarrow(X \wedge X)} x\right)\right), \\
K_{\forall X . X \Rightarrow(X \wedge X)}^{\prime \prime X} & \left.=K_{X \Rightarrow(X \wedge X)}^{\prime X}(0)^{\forall X . X \Rightarrow(X \wedge X)}[X]\right) \\
& =K_{X}^{X}\left(\pi_{X}\left((0)^{\forall X . X \Rightarrow(X \wedge X)}[X] x\right)\right) .
\end{aligned}
$$

Then,

$$
K_{\forall X . X \Rightarrow(X \wedge X)}^{\prime \prime X}\left(\Lambda X \cdot \lambda y^{X} \cdot\langle y, y\rangle\right)=\pi_{X}\left(\left(\Lambda X \cdot \lambda y^{X} \cdot\langle y, y\rangle\right)[X] x\right) .
$$

Definition 5.23 (Terms occurring in an elimination context). Let $K_{B}^{A}$ be an elimination context. The multiset of terms occurring in $K_{B}^{A}$ is defined as

$$
\begin{aligned}
\mathcal{T}\left(\left(0 D^{A}\right)\right. & =\emptyset \\
\mathcal{T}\left(K_{A}^{B \Rightarrow C} r\right) & =\mathcal{T}\left(K_{A}^{B \Rightarrow C}\right) \uplus\{r\} \\
\mathcal{T}\left(\pi_{B}\left(K_{A}^{B \wedge C}\right)\right) & =\mathcal{T}\left(K_{A}^{B \wedge C}\right) \\
\mathcal{T}\left(K_{A}^{\forall X . B}[C]\right) & =\mathcal{T}\left(K_{A}^{\forall X . B}\right)
\end{aligned}
$$

We write $\left|K_{A}^{B}\right|$ for $\sum_{i=1}^{n}\left|r_{i}\right|$ where $\left[r_{1}, \ldots, r_{n}\right]=\mathcal{T}\left(K_{A}^{B}\right)$.

Example 5.24. We have that $\left.\mathcal{T}(0)^{A} r s\right)=[r, s]$ and that $\left.\mathcal{T}(0)^{A}\langle r, s\rangle\right)=[\langle r, s\rangle]$. Remark that $K_{A}^{B}(t) \rightleftarrows *$ $K_{A}^{\prime B}(t)$ does not imply $\mathcal{T}\left(K_{A}^{B}\right) \sim \mathcal{T}\left(K_{A}^{\prime B}\right)$.

Definition 5.25 (Reducibility). The set of reducible terms of type $A$ (notation $\llbracket A \rrbracket$ ) is defined as the set of terms $r$ of type $A$ such that for any elimination context $K_{A}^{X}$ where all the terms in $\mathcal{T}\left(K_{A}^{X}\right)$ are in SN, we have $K_{A}^{X}(r) \in \mathrm{SN}$.

The following lemma is a trivial consequence of the definition of reducibility.

Lemma 5.26. For all $A, B$ such that $A \equiv B$, we have $\llbracket A \rrbracket=\llbracket B \rrbracket$.

Lemma 5.27. For all $A, \llbracket A \rrbracket \subseteq \mathrm{SN}$.

Proof. For all $A$, there exists an elimination context $K_{A}^{X}$, since variables are in SN and they can have any type. Hence, given that if $r \in \llbracket A \rrbracket$ then $K_{A}^{X}(r) \in \mathrm{SN}$, we have $r \in \mathrm{SN}$. 


\subsection{Adequacy}

We finally prove the adequacy theorem (Theorem 5.36) showing that every typed term is reducible, and the strong normalisation theorem (Theorem 5.37) as a consequence of it.

Lemma 5.28 (Adequacy of variables). For all $A$ and $x^{A}$, we have $x^{A} \in \llbracket A \rrbracket$.

Proof. We need to prove that $K_{A}^{X}(x) \in \mathrm{SN}$. The term $K_{A}^{X}(x)$ has the variable $x$ in a position that does not create any redex, hence the only redexes are those in $\mathcal{T}\left(K_{A}^{X}\right)$, which are already in SN. Then, $K_{A}^{X}(x) \in \mathrm{SN}$.

Lemma 5.29 (Adequacy of projection). For all $r, A, B$, such that $r \in \llbracket A \wedge B \rrbracket$, we have $\pi_{A}(r) \in \llbracket A \rrbracket$.

Proof. We need to prove that $K_{A}^{X}\left(\pi_{A}(r)\right) \in \mathrm{SN}$. Take $K_{A \wedge B}^{\prime X}=K_{A}^{X}\left(0 \pi_{A}\left(\left(D^{A \wedge B}\right)\right)\right.$, and since $r \in \llbracket A \wedge B \rrbracket$, we have $K_{A \wedge B}^{\prime X}(r)=K_{A}^{X}\left(\pi_{A}(r)\right) \in \mathrm{SN}$.

Lemma 5.30 (Adequacy of application). For all $r, s, A, B$ such that $r \in \llbracket A \Rightarrow B \rrbracket$ and $s \in \llbracket A \rrbracket$, we have $r s \in \llbracket B \rrbracket$.

Proof. We need to prove that $K_{B}^{X}(r s) \in \mathrm{SN}$. Take $\left.K_{A \Rightarrow B}^{\prime X}=K_{B}^{X}(0)^{A \Rightarrow B} s\right)$, and since $r \in \llbracket A \Rightarrow B \rrbracket$, we have $K_{A \Rightarrow B}^{\prime X}(r)=K_{B}^{X}(r s) \in \mathrm{SN}$.

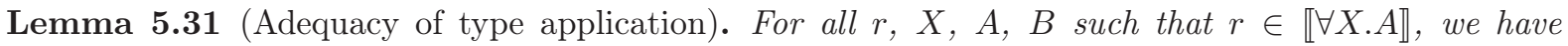
$r[B] \in \llbracket[X:=B] A \rrbracket$.

Proof. We need to prove that $K_{[X:=B] A}^{Y}(r[B]) \in \mathrm{SN}$. Take $K_{\forall X . A}^{Y Y}=K_{[X:=B] A}^{Y}\left(0 D^{\forall X . A}[B] D \in \mathrm{SN}\right.$, and since $r \in \llbracket \forall X . A \rrbracket$, we have $K_{\forall X . A}^{\prime Y}(r)=K_{[X:=B] A}^{Y}(r[B]) \in \mathrm{SN}$.

Lemma 5.32 (Adequacy of product). For all $r, s, A, B$ such that $r \in \llbracket A \rrbracket$ and $s \in \llbracket B \rrbracket$, we have $\langle r, s\rangle \in \llbracket A \wedge B \rrbracket$.

Proof. We need to prove that $K_{A \wedge B}^{X}\langle\langle r, s\rangle) \in \mathrm{SN}$. We proceed by induction on the number of projections in $K_{A \wedge B}^{X}$. Since the hole of $K_{A \wedge B}^{X}$ has type $A \wedge B$, and $K_{A \wedge B}^{X}(t)$ has type $X$ for any $t$ of type $A \wedge B$, we can assume, without lost of generality, that the context $K_{A \wedge B}^{X}$ has the form $K_{C}^{\prime X}\left(\pi_{C}\left(0 D^{A \wedge B} \alpha_{1} \ldots \alpha_{n}\right)\right)$, where each $\alpha_{i}$ is either a term or a type argument. We prove that all $K_{C}^{\prime X}\left(\pi_{C}\left(\left\langle r \alpha_{1} \ldots \alpha_{n}, s \alpha_{1} \ldots \alpha_{n}\right\rangle\right)\right) \in$ SN by showing, more generally, that if $r^{\prime}$ and $s^{\prime}$ are two reducts of $r \alpha_{1} \ldots \alpha_{n}$ and $s \alpha_{1} \ldots \alpha_{n}$, then $K_{C}^{\prime X}\left(\pi_{C}\left(\left\langle r^{\prime}, s^{\prime}\right\rangle\right) D \in \mathrm{SN}\right.$. For this, we show that all its one step reducts are in SN, by induction on $\left|K_{C}^{\prime X}\right|+\left|r^{\prime}\right|+\left|s^{\prime}\right|$. The full details are given in the technical appendix at [25].

Lemma 5.33 (Adequacy of abstraction). For all $t, r, x, A, B$ such that $t \in \llbracket A \rrbracket$ and $[x:=t] r \in \llbracket B \rrbracket$, we have $\lambda x^{A} . r \in \llbracket A \Rightarrow B \rrbracket$.

Proof. By induction on $M(r)$.

- If $r \rightleftarrows^{*}\left\langle r_{1}, r_{2}\right\rangle$, then by Lemma 4.12 , we have $B \equiv B_{1} \wedge B_{2}$ with $r_{1}$ of type $B_{1}$ and $r_{2}$ of type $B_{2}$, and so by Lemma $4.13,[x:=t] r_{1}$ has type $B_{1}$ and $[x:=t] r_{2}$ has type $B_{2}$. Since $[x:=t] r \in \llbracket B \rrbracket$, we have $\left\langle[x:=t] r_{1},[x:=t] r_{2}\right\rangle \in \llbracket B \rrbracket$. By Lemma 5.29, $[x:=t] r_{1} \in \llbracket B_{1} \rrbracket$ and $[x:=t] r_{2} \in \llbracket B_{2} \rrbracket$. By the induction hypothesis, $\lambda x^{A} \cdot r_{1} \in \llbracket A \Rightarrow B_{1} \rrbracket$ and $\lambda x^{A} \cdot r_{2} \in \llbracket A \Rightarrow B_{2} \rrbracket$, thus, by Lemma $5.32, \lambda x^{A} . r \rightleftarrows^{*}\left\langle\lambda x^{A} \cdot r_{1}, \lambda x^{A} \cdot r_{2}\right\rangle \in \llbracket\left(A \Rightarrow B_{1}\right) \wedge\left(A \Rightarrow B_{2}\right) \rrbracket$. Finally, by Lemma 5.26, we have $\llbracket\left(A \Rightarrow B_{1}\right) \wedge\left(A \Rightarrow B_{2}\right) \rrbracket=\llbracket A \Rightarrow B \rrbracket$.

- If $r \not \not^{*}\left\langle r_{1}, r_{2}\right\rangle$, we need to prove that for any elimination context $K_{A \Rightarrow B}^{X}$, we have $K_{A \Rightarrow B}^{X}(\Lambda X . r) \in$ SN. Since $r$ and all the terms in $\mathcal{T}\left(K_{A \Rightarrow B}^{X}\right)$ are in SN, we proceed by induction on the lexicographical order of $\left(\left|K_{A \Rightarrow B}^{X}\right|+|r|, M(r)\right)$ to show that all the one step reducts of $K_{A \Rightarrow B}^{X}\left(\lambda x^{A} . r\right)$ are in SN. Since $r$ is not a product, its only one step reducts are the following.

- A term where the reduction took place in one of the terms in $\mathcal{T}\left(K_{A \Rightarrow B}^{X}\right)$ or in $r$, and so we apply the induction hypothesis.

$-K_{B}^{\prime X}([x:=s] r)$, with $K_{A \Rightarrow B}^{X}\left(\lambda x^{A} . r\right)=K_{B}^{\prime X}\left[\left(\lambda x^{A} . r\right) s\right]$. Since $[x:=s] r \in \llbracket B \rrbracket$, we have $K_{B}^{\prime X}([x:=s] r D \in \mathrm{SN}$. 
- $K_{A \Rightarrow B^{\prime}}^{\prime X}\left(\lambda x^{A} \cdot[X:=C] r^{\prime}\right)$, with $r \rightleftarrows^{*}$-equivalent to $\Lambda X . r^{\prime}, B \equiv \forall X . B^{\prime}$, and $K_{A \Rightarrow B}^{X}\left(\lambda x^{A} . \Lambda X . r^{\prime}\right)$ is equal to $\left.K_{A \Rightarrow B^{\prime}}^{\prime X}\left(\lambda x^{A} \cdot \Lambda X . r^{\prime}\right)[C]\right)$. Since $M\left([X:=C] r^{\prime}\right)<M\left(\Lambda X . r^{\prime}\right)$, we apply the induction hypothesis.

Lemma 5.34 (Adequacy of type abstraction). For all $r, X, A, B$ such that $[X:=B] r \in \llbracket[X:=B] A \rrbracket$, we have $\Lambda X . r \in \llbracket \forall X . A \rrbracket$.

Proof. We proceed by induction on $M(r)$ with a proof similar to that of Lemma 5.33. Full details are given in the technical appendix at [25].

Definition 5.35 (Adequate substitution). A substitution $\theta$ is adequate for a context $\Gamma$ (notation $\theta \vDash \Gamma$ ) if for all $x: A \in \Gamma$, we have $\theta(x) \in \llbracket A \rrbracket$.

Theorem 5.36 (Adequacy). For all $\Gamma, r, A$, and substitution $\theta$ such that $\Gamma \vdash r: A$ and $\theta \vDash \Gamma$, we have $\theta r \in \llbracket A \rrbracket$.

Proof. By induction on $r$.

- If $r$ is a variable $x: A \in \Gamma$, then, since $\theta \vDash \Gamma$, we have $\theta r \in \llbracket A \rrbracket$.

- If $r$ is a product $\langle s, t\rangle$, then by Lemma 4.12, $\vdash \vdash: B, \Gamma \vdash t: C$, and $A \equiv B \wedge C$, thus, by the induction hypothesis, $\theta s \in \llbracket B \rrbracket$ and $\theta t \in \llbracket C \rrbracket$. By Lemma 5.32, $\langle\theta s, \theta t\rangle \in \llbracket B \wedge C \rrbracket$, hence by Lemma 5.26, $\theta r \in \llbracket A \rrbracket$.

- If $r$ is a projection $\pi_{A}(s)$, then by Lemma 4.12, $\Gamma \vdash s: A \wedge B$, and by the induction hypothesis, $\theta s \in \llbracket A \wedge B \rrbracket$. By Lemma 5.29, $\pi_{A}(\theta s) \in \llbracket A \rrbracket$, hence $\theta r \in \llbracket A \rrbracket$.

- If $r$ is an abstraction $\lambda x^{B} . s$, with $\Gamma \vdash s: C$, then by Lemma $4.12, A \equiv B \Rightarrow C$, hence by the induction hypothesis, for all $\theta$ and for all $t \in \llbracket B \rrbracket,[x:=t](\theta s) \in \llbracket C \rrbracket$. Hence, by Lemma 5.33, $\lambda x^{B} . \theta s \in \llbracket B \Rightarrow C \rrbracket$, so, by Lemma 5.26, $\theta r \in \llbracket A \rrbracket$.

- If $r$ is an application st, then by Lemma $4.12, \Gamma \vdash s: B \Rightarrow A$ and $\Gamma \vdash t: B$, thus, by the induction hypothesis, $\theta s \in \llbracket B \Rightarrow A \rrbracket$ and $\theta t \in \llbracket B \rrbracket$. Hence, by Lemma 5.30, we have $\theta r=\theta s \theta t \in \llbracket A \rrbracket$.

- If $r$ is a type abstraction $\Lambda X . s$, with $\Gamma \vdash s: B$, then by Lemma $4.12, A \equiv \forall X . B$, hence by the induction hypothesis, for all $\theta, \theta s \in \llbracket B \rrbracket$. Hence, by Lemma 5.34, $\Lambda X . \theta s \in \llbracket \forall X . B \rrbracket$, hence, by Lemma 5.26, $\theta r \in \llbracket A \rrbracket$.

- If $r$ is a type application $s[C]$, then by Lemma 4.12, $\Gamma \vdash s: \forall X . B$ with $A \equiv[X:=C] B$, thus, by the induction hypothesis, $\theta s \in \llbracket \forall X$. $B \rrbracket$. Hence, by Lemma 5.31, we have $\theta r=\theta s[C] \in \llbracket A \rrbracket$.

Theorem 5.37 (Strong normalisation). For all $\Gamma, r, A$ such that $\Gamma \vdash r: A$, we have $r \in \mathrm{SN}$.

Proof. By Lemma 5.27, the identity substitution is adequate. Thus, by Theorem 5.36 and Lemma 5.27, $r \in \llbracket A \rrbracket \subseteq \mathrm{SN}$.

\section{Conclusion, Discussion and Future Work}

System I is a simply-typed lambda calculus with pairs, extended with an equational theory obtained from considering the type isomorphisms as equalities. In this way, the system allows a programmer to focus on the meaning of programs, ignoring the rigid syntax of terms within the safe context provided by type isomorphisms. In this paper we have extended System I with polymorphism, and its corresponding isomorphisms, enriching the language with a feature that most programmers expect.

From a logical perspective, System I is a proof system for propositional logic, where isomorphic propositions have the same proofs, and PSI extends System I with the universal quantifier.

The main theorems in this paper prove subject reduction (Theorem 4.14) and strong normalisation (Theorem 5.37). The proof of the latter is a non-trivial adaptation of Girard's proof [20] for System F. 


\subsection{Swap}

As mentioned in Section 2, two isomorphisms for System F with pairs, as defined by Di Cosmo [9], are not considered explicitly: isomorphisms (7) and (8). However, the isomorphism (7) is just the $\alpha$-equivalence, which has been given implicitly, and so it has indeed been considered. The isomorphism that actually was not considered is (8), which allows to swap the type abstractions: $\forall X . \forall Y . A \equiv \forall Y . \forall X$.A. This isomorphism is the analogous to the isomorphism $A \Rightarrow B \Rightarrow C \equiv B \Rightarrow A \Rightarrow C$ at the first order level, which is a consequence of isomorphisms (4) and (1). At this first order level, the isomorphism induces the following equivalence:

$$
\begin{aligned}
\left(\lambda x^{A} \cdot \lambda y^{B} \cdot r\right) s t & \rightleftarrows\left(\lambda x^{A} \cdot \lambda y^{B} \cdot r\right)\langle s, t\rangle \\
& \rightleftarrows\left(\lambda x^{A} \cdot \lambda y^{B} \cdot r\right)\langle t, s\rangle \\
& \rightleftarrows\left(\lambda x^{A} \cdot \lambda y^{B} \cdot r\right) t s
\end{aligned}
$$

An alternative approach would have been to introduce an equivalence between $\lambda x^{A} \cdot \lambda y^{B} \cdot r$ and $\lambda y^{B} \cdot \lambda x^{A} \cdot r$. However, in any case, to keep subject reduction, the $\beta_{\lambda}$ reduction must verify that the type of the argument matches the type of the variable before reducing. This solution is not easily implementable for the $\beta_{\Lambda}$ reduction, since it involves using the type as a labelling for the term and the variable, to identify which term corresponds to which variable (leaving the posibility for non-determinism if the "labellings" are duplicated), but at the level of types we do not have a natural labelling.

Another alternative solution, in the same direction, is the one implemented by the selective lambda calculus [18], where only arrows, and not conjunctions, were considered, and so only the ismorphism $A \Rightarrow B \Rightarrow C \equiv B \Rightarrow A \Rightarrow C$ is treated. In the selective lambda calculus the solution is indeed to include external labellings (not types) to identify which argument is being used at each time. We could have added a labelling to type applications, $t\left[A_{X}\right]$, together with the following rule: $r\left[A_{X}\right]\left[B_{Y}\right] \rightleftarrows r\left[B_{Y}\right]\left[A_{X}\right]$ and so modifying the $\beta_{\Lambda}$ to $(\Lambda X . r)\left[A_{X}\right] \hookrightarrow[X:=A] r$.

Despite that such a solution seems to work, we found that it does not contribute to the language in any aspect, while it does make the system less readable. Therefore, we have decided to exclude the isomorphism (8) for PSI.

Another remark is that while a rule such as $r[A] \rightleftarrows r[B]$ with $A \equiv B$ seems to be admissible in the system, it may not be necessary. Indeed, the only rule that could benefit from it seems to be (P-DIST $\forall_{e} \wedge_{i}$ ) when used from right to left, which is only worthy when there is a $\Lambda$ that can be factorised, in which



\subsection{Future work}

\subsubsection{Eta-expansion rule}

An extended fragment of an early version [10] of System I has been implemented [15] in Haskell. In such an implementation, we have added some ad-hoc rules in order to have a progression property (that is, having only introductions as normal forms of closed terms). For example, "If $s$ has type $B$, then $\left(\lambda x^{A} \cdot \lambda y^{B} . r\right) s \hookrightarrow \lambda x^{A} .\left(\left(\lambda y^{B} \cdot r\right) s\right)$ ". Such a rule, among others introduced in this implementation, is a particular case of a more general $\eta$-expansion rule. Certainly, with the rule $r \hookrightarrow \lambda x^{A}$.rx we can derive

$$
\begin{aligned}
\left(\lambda x^{A} \cdot \lambda y^{B} \cdot r\right) s & \hookrightarrow \lambda z^{A} \cdot\left(\lambda x^{A} \cdot \lambda y^{B} \cdot r\right) s z \\
& \rightleftarrows^{*} \lambda z^{A} \cdot\left(\lambda x^{A} \cdot \lambda y^{B} \cdot r\right) z s \\
& \hookrightarrow \lambda z^{A} \cdot\left(\left(\lambda y^{B} \cdot[x:=z] r\right) s\right)
\end{aligned}
$$

In [13] we have showed that it is indeed the case that all the ad-hoc rules from [10] can be lifted by adding extensional rules.

In addition, the proof of the consistency of PSI as a language of proof-terms for second-order logic has been intentionally left out of this paper. Indeed, as shown in [12], it would require to restrict variables to only have "prime types", that is non-conjunctive types. Such a restriction has also been shown to be not necessary when the language is extended with eta rules [13]. Therefore, we preferred to delay the proof of consistency for a future version of PSI with $\eta$-rules. 


\subsubsection{Implementation}

The mentioned implementation of an early version of System I, included a fix point operator and numbers, showing some interesting programming examples. We plan to extend such an implementation for polymorphism, following the design of PSI.

\section{References}

[1] Pablo Arrighi and Alejandro Díaz-Caro. A System F accounting for scalars. LMCS, 8(1:11):1-32, 2012.

[2] Pablo Arrighi, Alejandro Díaz-Caro, and Benoît Valiron. The vectorial lambda-calculus. Inf. and Comp., 254(1):105-139, 2017.

[3] Pablo Arrighi and Gilles Dowek. Lineal: A linear-algebraic lambda-calculus. LMCS, 13(1:8):1-33, 2017.

[4] Gérard Boudol. Lambda-calculi for (strict) parallel functions. Inf. and Comp., 108(1):51-127, 1994.

[5] Antonio Bucciarelli, Thomas Ehrhard, and Giulio Manzonetto. A relational semantics for parallelism and non-determinism in a functional setting. APAL, 163(7):918-934, 2012.

[6] Thierry Coquand and Gérard Huet. The calculus of constructions. Inf. and Comp., 76(2-3):95-120, 1988.

[7] Ugo de'Liguoro and Adolfo Piperno. Non deterministic extensions of untyped $\lambda$-calculus. Inf. and Comp., 122(2):149-177, 1995.

[8] Mariangiola Dezani-Ciancaglini, Ugo de'Liguoro, and Adolfo Piperno. A filter model for concurrent $\lambda$-calculus. SIAM JComp., 27(5):1376-1419, 1998.

[9] Roberto Di Cosmo. Isomorphisms of types: from $\lambda$-calculus to information retrieval and language design. Progress in Theoretical Computer Science. Birkhauser, Switzerland, 1995.

[10] Alejandro Díaz-Caro and Gilles Dowek. Non determinism through type isomorphism. EPTCS (LSFA'12), 113:137-144, 2013.

[11] Alejandro Díaz-Caro and Gilles Dowek. Typing quantum superpositions and measurement. LNCS (TPNC'17), 10687:281-293, 2017.

[12] Alejandro Díaz-Caro and Gilles Dowek. Proof normalisation in a logic identifying isomorphic propositions. LIPIcs (FSCD'19), 131:14:1-14:23, 2019.

[13] Alejandro Díaz-Caro and Gilles Dowek. Extensional proofs in a propositional logic modulo isomorphisms. arXiv:2002.03762, 2020.

[14] Alejandro Díaz-Caro, Mauricio Guillermo, Alexandre Miquel, and Benoît Valiron. Realizability in the unitary sphere. In Proceedings of the 34th Annual ACM/IEEE Symposium on Logic in Computer Science (LICS 2019), pages 1-13, Vancouver, BC, Canada, 2019. IEEE.

[15] Alejandro Díaz-Caro and Pablo E. Martínez López. Isomorphisms considered as equalities: Projecting functions and enhancing partial application through an implementation of $\lambda^{+}$. ACM IFL, 2015(9):1-11, 2015.

[16] Gilles Dowek, Thérèse Hardin, and Claude Kirchner. Theorem proving modulo. JAR, 31(1):33-72, 2003.

[17] Gilles Dowek and Benjamin Werner. Proof normalization modulo. JSL, 68(4):1289-1316, 2003.

[18] Jacques Garrigue and Hassan Aït-Kaci. The typed polymorphic label-selective $\lambda$-calculus. In Proceedings of the 21st ACM SIGPLAN-SIGACT Symposium on Principles of Programming Languages, POPL '94, page 35-47, New York, NY, USA, 1994. Association for Computing Machinery. 
[19] Herman Geuvers, Robbert Krebbers, James McKinna, and Freek Wiedijk. Pure type systems without explicit contexts. In Karl Crary and Marino Miculan, editors, Proceedings of LFMTP 2010, volume 34 of EPTCS, pages 53-67, 2010.

[20] Jean-Yves Girard, Paul Taylor, and Yves Lafont. Proofs and types. Cambridge U.P., UK, 1989.

[21] Per Martin-Löf. Intuitionistic type theory. Bibliopolis, Napoli, Italy, 1984.

[22] Michele Pagani and Simona Ronchi Della Rocca. Linearity, non-determinism and solvability. Fund. Inf., 103(1-4):173-202, 2010.

[23] Jonghyun Park, Jeongbong Seo, Sungwoo Park, and Gyesik Lee. Mechanizing metatheory without typing contexts. Journal of Automated Reasoning, 52(2):215-239, 2014.

[24] Mikael Rittri. Retrieving library identifiers via equational matching of types. In Proceedings of CADE 1990, volume 449 of LNCS, pages 603-617, 1990.

[25] Cristian F. Sottile, Alejandro Díaz-Caro, and Pablo E. Martínez López. Polymorphic system i. arXiv:2101.03215, 2021.

[26] The Univalent Foundations Program. HoTT: Univalent Foundations of Mathematics. Institute for Advanced Study, Princeton, NJ, USA, 2013.

[27] Lionel Vaux. The algebraic lambda calculus. MSCS, 19(5):1029-1059, 2009. 


\section{A Detailed proofs of Section 4}

Lemma 4.9. For all $X, A, B, C$ such that $\forall X . A \equiv B \wedge C$, there exist $B^{\prime}, C^{\prime}$ such that $B \equiv \forall X . B^{\prime}$, $C \equiv \forall X . C^{\prime}$ and $A \equiv B^{\prime} \wedge C^{\prime}$.

Proof. By Lemma 4.5, $P F(\forall X . A) \sim P F(B \wedge C)=P F(B) \uplus P F(C)$.

By Lemma 4.2, let $P F(A)=\left[\forall \vec{Y}_{i} \cdot\left(A_{i} \Rightarrow Z_{i}\right)\right]_{i=1}^{n}, P F(B)=\left[\forall \vec{W}_{j} \cdot\left(D_{j} \Rightarrow Z_{j}^{\prime}\right)\right]_{j=1}^{k}$, and $P F(C)=$ $\left[\forall \vec{W}_{j} \cdot\left(D_{j} \Rightarrow Z_{j}^{\prime}\right)\right]_{j=k+1}^{m}$.

Hence, $\left[\forall X . \forall \vec{Y}_{i} .\left(A_{i} \Rightarrow Z_{i}\right)\right]_{i=1}^{n} \sim\left[\forall \vec{W}_{j} .\left(D_{j} \Rightarrow Z_{j}^{\prime}\right)\right]_{j=1}^{m}$. So, by definition of $\sim, n=m$ and for $i=1, \ldots, n$ and a permutation $p$, we have $\forall X . \forall \vec{Y}_{i} \cdot\left(A_{i} \Rightarrow Z_{i}\right) \equiv \forall \vec{W}_{p(i)} \cdot\left(D_{p(i)} \Rightarrow Z_{p(i)}^{\prime}\right)$. Thus, by Lemma 4.7, we have $X, \vec{Y}_{i}=\vec{W}_{p(i)}, A_{i} \equiv D_{p(i)}$, and $Z_{i}=Z_{p(i)}^{\prime}$. Therefore, there exists $I$ such that $I \cup \bar{I}=$ $\{1, \ldots, n\}$, such that $P F(B)=\left[\forall \vec{W}_{p(i)} \cdot\left(D_{p(i)} \Rightarrow Z_{p(i)}^{\prime}\right)\right]_{i \in I}$ and $P F(C)=\left[\forall \vec{W}_{p(i)} \cdot\left(D_{p(i)} \Rightarrow Z_{p(i)}^{\prime}\right)\right]_{i \in \bar{I}}$. Hence, by Corollary 4.3 , we have, $B \equiv \bigwedge_{i \in I} \forall \vec{W}_{p(i)} \cdot\left(D_{p(i)} \Rightarrow Z_{p_{i}}^{\prime}\right) \equiv \bigwedge_{i \in I} \forall X . \forall \vec{Y}_{i} \cdot\left(A_{i} \Rightarrow Z_{i}\right)$ and $C \equiv$ $\bigwedge_{i \in \bar{I}} \forall X . \forall \vec{Y}_{i} \cdot\left(A_{i} \Rightarrow Z_{i}\right)$.

Let $B^{\prime}=\bigwedge_{i \in I} \forall \vec{Y}_{i} .\left(A_{i} \Rightarrow Z_{i}\right)$ and $C^{\prime}=\bigwedge_{i \in \bar{I}} \forall \vec{Y}_{i} .\left(A_{i} \Rightarrow Z_{i}\right)$. So, $B \equiv \forall X . B^{\prime}$ and $C \equiv \forall X . C^{\prime}$. Hence, also by Corollary 4.3, we have $A \equiv \bigwedge_{i=1}^{n} \forall \vec{Y}_{i} \cdot\left(A_{i} \Rightarrow Z_{i}\right) \equiv B^{\prime} \wedge C^{\prime}$.

Lemma 4.10. For all $X, A, B, C$ such that $\forall X . A \equiv B \Rightarrow C$, there exists $C^{\prime}$ such that $C \equiv \forall X . C^{\prime}$ and $A \equiv B \Rightarrow C^{\prime}$.

Proof. By Lemma 4.5, $P F(\forall X . A) \sim P F(B \Rightarrow C)$.

By Lemma 4.2, let $P F(A)=\left[\forall \vec{Y}_{i} \cdot\left(A_{i} \Rightarrow Z_{i}\right)\right]_{i=1}^{n}$ and $P F(C)=\left[\forall \vec{W}_{j} \cdot\left(D_{j} \Rightarrow Z_{j}^{\prime}\right)\right]_{j=1}^{m}$. Hence, $\left[\forall X . \forall \vec{Y}_{i} \cdot\left(A_{i} \Rightarrow Z_{i}\right)\right]_{i=1}^{n} \sim\left[\forall \vec{W}_{j} .\left(\left(B \wedge D_{j}\right) \Rightarrow Z_{j}^{\prime}\right)\right]_{j=1}^{m}$. So, by definition of $\sim, n=m$ and for $i=1, \ldots, n$ and a permutation $p$, we have $\forall X . \forall \vec{Y}_{i} \cdot\left(A_{i} \Rightarrow Z_{i}\right) \equiv \forall \vec{W}_{p(i)} \cdot\left(\left(B \wedge D_{p(i)}\right) \Rightarrow Z_{p(i)}^{\prime}\right)$

Hence, by Lemma 4.7, we have $X, \vec{Y}_{i}=\vec{W}_{p(i)}, A_{i} \equiv B \wedge D_{p(i)}$, and $Z_{i}=Z_{p(i)}^{\prime}$. Hence, by Corollary 4.3,

$$
C \equiv \bigwedge_{j=1}^{n} \forall \vec{W}_{j} \cdot\left(D_{j} \Rightarrow Z_{j}^{\prime}\right) \equiv \bigwedge_{i=1}^{n} \forall \vec{W}_{p(i)} .\left(D_{p(i)} \Rightarrow Z_{p(i)}^{\prime}\right) \equiv \bigwedge_{i=1}^{n} \forall X . \forall \vec{Y}_{i} \cdot\left(D_{p(i)} \Rightarrow Z_{i}\right)
$$

Let $C^{\prime}=\bigwedge_{i=1}^{n} \forall \vec{Y}_{i} .\left(D_{p(i)} \Rightarrow Z_{i}\right)$. So, $C \equiv \forall X . C^{\prime}$.

Hence, also by Corollary 4.3 , we have

$$
A \equiv \bigwedge_{i=1}^{n} \forall \vec{Y}_{i} \cdot\left(A_{i} \Rightarrow Z_{i}\right) \equiv \bigwedge_{i=1}^{n} \forall \vec{Y}_{i} \cdot\left(\left(B \wedge D_{p(i)}\right) \Rightarrow Z_{i}\right) \equiv B \Rightarrow \bigwedge_{i=1}^{n} \forall \vec{Y}_{i} \cdot\left(D_{p(i)} \Rightarrow Z_{i}\right) \equiv B \Rightarrow C^{\prime}
$$

Lemma 4.11 (Unicity modulo). For all $\Gamma, r, A, B$ such that $\Gamma \vdash r: A$ and $\Gamma \vdash r: B$, we have $A \equiv B$.

Proof.

- If the last rule of the derivation of $\Gamma \vdash r: A$ is $(\equiv)$, then we have a shorter derivation of $\Gamma \vdash r: C$ with $C \equiv A$, and, by the induction hypothesis, $C \equiv B$, hence $A \equiv B$.

- If the last rule of the derivation of $\Gamma \vdash r: B$ is (三) we proceed in the same way.

- All the remaining cases are syntax directed.

Lemma 4.13 (Substitution).

1. For all $\Gamma, x, r, s, A, B$ such that $\Gamma, x: B \vdash r: A$ and $\Gamma \vdash s: B$, we have $\Gamma \vdash[x:=s] r: A$.

2. For all $\Gamma, r, X, A, B$ such that $\Gamma \vdash r: A$, we have $[X:=B] \Gamma \vdash[X:=B] r:[X:=B] A$.

Proof.

1. By structural induction on $r$. 
- Let $r=x$. By Lemma 4.12, $A \equiv B$, thus $\Gamma \vdash s: A$. Since $[x:=s] x=s$, we have $\Gamma \vdash[x:=s] x: A$.

- Let $r=y$, with $y \neq x$. Since $[x:=s] y=y$, we have $\Gamma \vdash[x:=s] y: A$.

- Let $r=\lambda x^{C}$.t. We have $[x:=s]\left(\lambda x^{C} . t\right)=\lambda x^{C}$.t, so $\Gamma \vdash[x:=s]\left(\lambda x^{C} . t\right): A$.

- Let $r=\lambda y^{C}$.t, with $y \neq x$. By Lemma 4.12, $A \equiv C \Rightarrow D$ and $\Gamma, y: C \vdash t: D$. By the induction hypothesis, $\Gamma, y: C \vdash[x:=s] t: D$, and so, by rule $\left(\Rightarrow_{i}\right), \Gamma \vdash \lambda y^{C} \cdot[x:=s] t: C \Rightarrow D$. Since $\lambda y^{C} \cdot[x:=s] t=[x:=s]\left(\lambda y^{C} . t\right)$, using rule $(\equiv), \Gamma \vdash[x:=s]\left(\lambda x^{C} . t\right): A$.

- Let $r=t u$. By Lemma 4.12, $\Gamma \vdash t: C \Rightarrow A$ and $\Gamma \vdash u: C$. By the induction hypothesis, $\Gamma \vdash$ $[x:=s] t: C \Rightarrow A$ and $\Gamma \vdash[x:=s] u: C$, and so, by rule $\left(\Rightarrow_{e}\right), \Gamma \vdash([x:=s] t)([x:=s] u): A$. Since $([x:=s] t)([x:=s] u)=[x:=s](t u)$, we have $\Gamma \vdash[x:=s](t u): A$.

- Let $r=\langle t, u\rangle$. By Lemma 4.12, $\Gamma \vdash t: C$ and $\Gamma \vdash u: D$, with $A \equiv C \wedge D$. By the induction hypothesis, $\Gamma \vdash[x:=s] t: C$ and $\Gamma \vdash[x:=s] u: D$, and so, by rule $\left(\wedge_{i}\right)$, $\Gamma \vdash\langle[x:=s] t,[x:=s] u\rangle: C \wedge D$. Since $\langle[x:=s] t,[x:=s] u\rangle=[x:=s]\langle t, u\rangle$, using rule $(\equiv)$, we have $\Gamma \vdash[x:=s]\langle t, u\rangle: A$.

- Let $r=\pi_{A}(t)$. By Lemma 4.12, $\Gamma \vdash t: A \wedge C$. By the induction hypothesis, $\Gamma \vdash[x:=s] t$ : $A \wedge C$, and so, by rule $\left(\wedge_{e}\right), \Gamma \vdash \pi_{A}([x:=s] t): A$. Since $\pi_{A}([x:=s] t)=[x:=s]\left(\pi_{A}(t)\right)$, we have $\Gamma \vdash[x:=s]\left(\pi_{A}(t)\right): A$.

- Let $r=\Lambda X$.t. By Lemma 4.12, $A \equiv \forall X$.C and $\Gamma \vdash t: C$. By the induction hypothesis, $\Gamma \vdash[x:=s] t: C$, and so, by rule $\left(\forall_{i}\right), \Gamma \vdash \Lambda X .[x:=s] t: \forall X$. C. Since $\Lambda X .[x:=s] t=$ $[x:=s](\Lambda X . t)$, using rule $(\equiv)$, we have $\Gamma \vdash[x:=s](\Lambda X . t): A$.

- Let $r=t[C]$. By Lemma 4.12, $A \equiv[X:=C] D$ and $\Gamma \vdash t: \forall X$.D. By the induction hypothesis, $\Gamma \vdash[x:=s] t: \forall X . D$, and so, by rule $\left(\forall_{e}\right), \Gamma \vdash([x:=s] t)[C]:[X:=C] D$. Since $([x:=s] t)[C]=[x:=s](t[C])$, using rule $(\equiv)$, we have $\Gamma \vdash[x:=s](t[C]): A$.

2. By induction on the typing relation.

- (ax): Let $\Gamma, x: A \vdash x: A$. Then, using rule (ax), we have $[X:=B] \Gamma, x:[X:=B] A \vdash[X:=$ $B] x:[X:=B] A$.

- $(\equiv)$ : Let $\Gamma \vdash r: A$, with $A \equiv C$. By the induction hypothesis, $[X:=B] \Gamma \vdash[X:=$ $B] r:[X:=B] C$. Since $A \equiv C,[X:=B] A \equiv[X:=B] C$. Using rule (三), we have $[X:=B] \Gamma \vdash[X:=B] r:[X:=B] A$.

- $\left(\Rightarrow_{i}\right)$ : Let $\Gamma \vdash \lambda x^{C} . t: C \Rightarrow D$. By the induction hypothesis, $[X:=B] \Gamma, x:[X:=B] C \vdash$ $[X:=B] t:[X:=B] D$. Using rule $\left(\Rightarrow_{i}\right),[X:=B] \Gamma \vdash \lambda x^{[X:=B] C} \cdot[X:=B] t:[X:=B] C \Rightarrow$ $[X:=B] D$. Since $\lambda x^{[X:=B] C}$. $[X:=B] t=[X:=B]\left(\lambda x^{C} . t\right)$, we have $[X:=B] \Gamma \vdash[X:=$ $B]\left(\lambda x^{C} . t\right):[X:=B](C \Rightarrow D)$.

- $\left(\Rightarrow_{e}\right)$ : Let $\Gamma \vdash t s: D$. By the induction hypothesis, $[X:=B] \Gamma \vdash[X:=B] t:[X:=B](C \Rightarrow$ $D)$ and $[X:=B] \Gamma \vdash[X:=B] s:[X:=B] C$. Since $[X:=B](C \Rightarrow D)=[X:=B] C \Rightarrow[X:=$ $B] D$, using rule $\left(\Rightarrow_{e}\right)$, we have $[X:=B] \Gamma \vdash([X:=B] t)([X:=B] s):[X:=B] D$. Since $([X:=B] t)([X:=B] s)=[X:=B](t s)$, we have $[X:=B] \Gamma \vdash[X:=B](t s):[X:=B] D$.

- $\left(\wedge_{i}\right)$ : Let $\Gamma \vdash\langle t, s\rangle: C \wedge D$. By the induction hypothesis, $[X:=B] \Gamma \vdash[X:=B] t:[X:=B] C$ and $[X:=B] \Gamma \vdash[X:=B] s:[X:=B] D$. Using rule $\left(\wedge_{i}\right),[X:=B] \Gamma \vdash\langle[X:=B] t,[X:=$ $B] s\rangle:[X:=B] C \wedge[X:=B] D$. Since $\langle[X:=B] t,[X:=B] s\rangle=[X:=B]\langle t, s\rangle$, and $[X:=$ $B] C \wedge[X:=B] D=[X:=B](C \wedge D)$, we have $[X:=B] \Gamma \vdash[X:=B]\langle t, s\rangle:[X:=B](C \wedge D)$.

- $\left(\wedge_{e}\right)$ : Let $\Gamma \vdash t: C \wedge D$. By the induction hypothesis, $[X:=B] \Gamma \vdash[X:=B] t:[X:=$ $B](C \wedge D)$. Since $[X:=B](C \wedge D)=[X:=B](C) \wedge[X:=B](D)$, using rule $\left(\wedge_{e}\right)$ we have $[X:=B] \Gamma \vdash \pi_{[X:=B] C}([X:=B] t):[X:=B](C)$. Since $\pi_{[X:=B] C}([X:=B] t)=$ $[X:=B] \pi_{C}(t)$, we have $[X:=B] \Gamma \vdash[X:=B] \pi_{C}(t):[X:=B](C)$.

- $\left(\forall_{i}\right)$ : Let $\Gamma \vdash \Lambda Y$.t $: \forall Y$.C, with $X \notin F T V(\Gamma)$. By the induction hypothesis, $[X:=B] \Gamma \vdash$ $[X:=B] t:[X:=B] C$. Since $X \notin F T V(\Gamma), X \notin F V([X:=B] \Gamma)$. Using rule $\left(\forall_{i}\right)$, we have $[X:=B] \Gamma \vdash \Lambda Y$. $[X:=B] t: \Lambda Y$. $[X:=B] C$. Since $\Lambda Y$. $[X:=B] t=[X:=B] \Lambda Y$.t, and $\forall Y$. $[X:=B] C=[X:=B] \forall Y$.C , we have $[X:=B] \Gamma \vdash[X:=B] \Lambda Y . t:[X:=B] \forall Y . C$. 
- $\left(\forall_{e}\right)$ : Let $\Gamma \vdash t[D]:[Y:=D] C$. By the induction hypothesis, $[X:=B] \Gamma \vdash[X:=B] t:[X:=$ $B] \forall Y$.C. Since $[X:=B] \forall Y . C=\forall Y$. $[X:=B] C$, using rule $\left(\forall_{e}\right)$, we have $[X:=B] \Gamma \vdash([X:=$ $B] t)[[X:=B] D]:[Y:=[X:=B] D][X:=B] C$.

Since $([X:=B] t)[[X:=B] D]=[X:=B](t[D])$, and $[Y:=[X:=B] D][X:=B] C=$ $[X:=B][Y:=D] C$, we have $[X:=B] \Gamma \vdash[X:=B](t[D]):[X:=B][Y:=D] C$.

Theorem 4.14 (Subject reduction). For all $\Gamma, r, s, A$ such that $\Gamma \vdash r: A$ and $r \hookrightarrow s$ or $r \rightleftarrows s$, we have $\Gamma \vdash s: A$.

Proof. By induction on the rewrite relation.

(сомм): $\langle t, r\rangle \rightleftarrows\langle r, t\rangle$

$(\rightarrow)$

1. $\Gamma \vdash\langle t, r\rangle: A$

(Hypothesis)

2. $A \equiv B \wedge C$

$\Gamma \vdash t: B$

$\Gamma \vdash r: C$

(1, Lemma 4.12)

3. $B \wedge C \equiv C \wedge B$

(Iso. (1))

4.

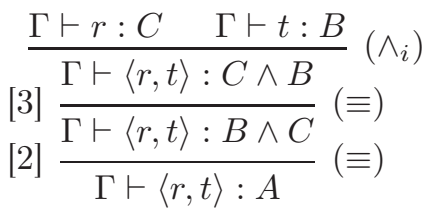

$(\leftarrow)$ analogous to $(\rightarrow)$.

(Asso): $\langle t,\langle r, s\rangle\rangle \rightleftarrows\langle\langle t, r\rangle, s\rangle$

$(\rightarrow)$

1. $\Gamma \vdash\langle t,\langle r, s\rangle\rangle: A$

(Hypothesis)

2. $A \equiv B \wedge C$

$\Gamma \vdash t: B$

$\Gamma \vdash\langle r, s\rangle: C$

(1, Lemma 4.12)

3. $C \equiv D \wedge E$

$\Gamma \vdash r: D$

$\Gamma \vdash s: E$

(2, Lemma 4.12)

(Iso. (2))

4. $B \wedge(D \wedge E) \equiv(B \wedge D) \wedge E$

$(2,3$, congr. ( $\equiv))$

5. $A \equiv B \wedge(D \wedge E)$

6.

$$
\begin{aligned}
& \frac{\Gamma \vdash t: B \quad \Gamma \vdash r: D}{\Gamma \vdash\langle t, r\rangle: B \wedge D}\left(\wedge_{i}\right) \quad \Gamma \vdash s: E \\
& \quad[4] \frac{\Gamma \vdash\langle\langle t, r\rangle, s\rangle:(B \wedge D) \wedge E}{\Gamma \vdash\langle\langle t, r\rangle, s\rangle: B \wedge(D \wedge E)}(\equiv) \\
& {[5] \frac{\Gamma \vdash\langle\langle t, r\rangle, s\rangle: A}{\Gamma}\left(\wedge_{i}\right)}
\end{aligned}
$$

$(\leftarrow)$ analogous to $(\rightarrow)$.

$\left(\operatorname{DIST}_{\lambda}\right): \lambda x^{A} \cdot\langle t, r\rangle \rightleftarrows\left\langle\lambda x^{A} . t, \lambda x^{A} \cdot r\right\rangle$

$(\rightarrow)$

1. $\Gamma \vdash \lambda x^{A} .\langle t, r\rangle: B$

(Hypothesis)

2. $B \equiv A \Rightarrow C$

$\Gamma, x: A \vdash\langle t, r\rangle: C$

(1, Lemma 4.12) 
3. $C \equiv D \wedge E$

$\Gamma, x: A \vdash t: D$

$\Gamma, x: A \vdash r: E$

4. $A \Rightarrow(D \wedge E) \equiv(A \Rightarrow D) \wedge(A \Rightarrow E)$

(2, Lemma 4.12)

5. $B \equiv A \Rightarrow(D \wedge E)$

6.

$$
\frac{\frac{\Gamma, x: A \vdash t: D}{\Gamma \vdash \lambda x^{A} \cdot t: A \Rightarrow D}\left(\Rightarrow_{i}\right) \quad \frac{\Gamma, x: A \vdash r: E}{\Gamma \vdash \lambda x^{A} \cdot r: A \Rightarrow E}}{[4] \frac{\Gamma \vdash\left\langle\lambda x^{A} \cdot t, \lambda x^{A} \cdot r\right\rangle:(A \Rightarrow D) \wedge(A \Rightarrow E)}{[5] \frac{\Gamma \vdash\left\langle\lambda x^{A} \cdot t, \lambda x^{A} \cdot r\right\rangle: A \Rightarrow(D \wedge E)}{\Gamma \vdash\left\langle\lambda x^{A} \cdot t, \lambda x^{A} \cdot r\right\rangle: B}(\equiv)}\left(\Rightarrow_{i}\right)}(\equiv)
$$

$(\leftarrow)$

1. $\Gamma \vdash\left\langle\lambda x^{A} . t, \lambda x^{A} . r\right\rangle: B$

2. $B \equiv C \wedge D$

$\Gamma \vdash \lambda x^{A} . t: C$

$\Gamma \vdash \lambda x^{A} . r: D$

(1, Lemma 4.12)

3. $C \equiv A \Rightarrow C^{\prime}$

$\Gamma, x: A \vdash t: C^{\prime}$

4. $D \equiv A \Rightarrow D^{\prime}$

$\Gamma, x: A \vdash r: D^{\prime}$

(2, Lemma 4.12)

(2, Lemma 4.12)

(Iso. (3))

5. $\left(A \Rightarrow C^{\prime}\right) \wedge\left(A \Rightarrow D^{\prime}\right) \equiv A \Rightarrow\left(C^{\prime} \wedge D^{\prime}\right)$

$(2,3,4$, congr. (三))

6. $B \equiv\left(A \Rightarrow C^{\prime}\right) \wedge\left(A \Rightarrow D^{\prime}\right)$

7.

$\frac{\Gamma, x: A \vdash t: C^{\prime} \quad \Gamma, x: A \vdash r: D^{\prime}}{\Gamma, x: A \vdash\langle t, r\rangle: C^{\prime} \wedge D^{\prime}}\left(\wedge_{i}\right)$
$[5] \frac{\frac{{ }^{\prime}{ }^{\prime} \lambda x^{A} \cdot\langle t, r\rangle: A \Rightarrow\left(C^{\prime} \wedge D^{\prime}\right)}{\Gamma \vdash \lambda x^{A} \cdot\langle t, r\rangle:\left(A \Rightarrow C^{\prime}\right) \wedge\left(A \Rightarrow D^{\prime}\right)}}{\Gamma \vdash \lambda x^{A} \cdot\langle t, r\rangle: B}$
$[6] \frac{(\equiv)}{(\equiv)}$

$\left(\mathrm{DIST}_{\mathrm{app}}\right):\langle t, r\rangle s \rightleftarrows\langle t s, r s\rangle$

$(\rightarrow)$

1. $\Gamma \vdash\langle t, r\rangle s: A$

(Hypothesis)

2. $\Gamma \vdash\langle t, r\rangle: B \Rightarrow A$

$\Gamma \vdash s: B$

(1, Lemma 4.12)

3. $B \Rightarrow A \equiv C \wedge D$

$\Gamma \vdash t: C$

$\Gamma \vdash r: D$

(2, Lemma 4.12)

4. $C \equiv B \Rightarrow C^{\prime}$

$D \equiv B \Rightarrow D^{\prime}$

$A \equiv C^{\prime} \wedge D^{\prime}$

(3, Lemma 4.8)

5.

6.

$$
\text { [4] } \frac{\frac{\Gamma \vdash t: C}{\Gamma \vdash t: B \Rightarrow C^{\prime}}(\equiv) \quad \Gamma \vdash s: B}{\Gamma \vdash t s: C^{\prime}}\left(\Rightarrow_{e}\right)
$$

7.

$$
\text { [4] } \frac{\frac{\Gamma \vdash r: D}{\Gamma \vdash r: B \Rightarrow D^{\prime}}(\equiv) \quad \Gamma \vdash s: B}{\Gamma \vdash r s: D^{\prime}}\left(\Rightarrow_{e}\right)
$$




$$
\text { [4] } \frac{\frac{(5)}{\Gamma \vdash t s: C^{\prime}} \frac{(6)}{\Gamma \vdash\langle t s, r s\rangle: C^{\prime} \wedge D^{\prime}}}{\Gamma \vdash\langle t s, r s\rangle: A}(\equiv)
$$

$(\leftarrow)$
1. $\Gamma \vdash\langle t s, r s\rangle: A$
2. $A \equiv B \wedge C$
$\Gamma \vdash t s: B$
$\Gamma \vdash r s: C$
3. $\begin{array}{r}\Gamma \vdash t: D \Rightarrow B \\ \Gamma \vdash s: D\end{array}$
4. $\Gamma \vdash r: E \Rightarrow B$
$\Gamma \vdash s: E$
5. $D \equiv E$
6. $D \Rightarrow(B \wedge C) \equiv(D \Rightarrow B) \wedge(D \Rightarrow C)$
7. $E \Rightarrow C \equiv D \Rightarrow C$
(Hypothesis)
(1, Lemma 4.12)
(2, Lemma 4.12)
(2, Lemma 4.12)
(3, 4, Lemma 4.11)
(Iso. (3))
$(6$, congr. $(\equiv))$

8.

$$
\begin{gathered}
\frac{\Gamma \vdash t: D \Rightarrow B \quad[7] \frac{\Gamma \vdash r: E \Rightarrow C}{\Gamma \vdash r: D \Rightarrow C}}{[5] \frac{\Gamma \vdash\langle t, r\rangle:(D \Rightarrow B) \wedge(D \Rightarrow C)}{\Gamma \vdash\langle t, r\rangle: D \Rightarrow(B \wedge C)}}\left(\begin{array}{l}
\left(\wedge_{i}\right) \\
\frac{\Gamma \vdash\langle t, r\rangle s: B \wedge C}{\Gamma \vdash\langle t, r\rangle s: A}(\equiv)
\end{array}\left({ }_{e}\right)\right. \\
\left.[2] \frac{}{\Gamma}\right)
\end{gathered}
$$

(CURRY): $t\langle r, s\rangle \rightleftarrows t r s$

$(\rightarrow)$

1. $\Gamma \vdash t\langle r, s\rangle: A$

2. $\Gamma \vdash t: B \Rightarrow A$

$\Gamma \vdash\langle t, r\rangle: B$

3. $B \equiv C \wedge D$

$\Gamma \vdash r: C$

$\Gamma \vdash s: D$

4. $B \Rightarrow A \equiv(C \wedge D) \Rightarrow A$

5. $(C \wedge D) \Rightarrow A \equiv C \Rightarrow(D \Rightarrow A)$

6.

$$
\begin{aligned}
& {[4] \frac{\Gamma \vdash t: B \Rightarrow A}{\Gamma \vdash t:(C \wedge D) \Rightarrow A}(\equiv)} \\
& {\left[\frac{\frac{\Gamma \vdash t: C \Rightarrow(D \Rightarrow A)}{\Gamma \vdash t} \quad \Gamma \vdash r: C}{\Gamma \vdash t r: D \Rightarrow A}\left(\Rightarrow_{e}\right)\right.}
\end{aligned}
$$

7.

$$
\frac{\frac{(6)}{\Gamma \vdash t r: D \Rightarrow A} \quad \Gamma \vdash s: D}{\Gamma \vdash t r s: A}\left(\Rightarrow_{e}\right)
$$

$(\leftarrow)$

$$
\begin{array}{ll}
\text { 1. } & \Gamma \vdash \operatorname{trs}: A \\
\text { 2. } & \Gamma \vdash t r: B \Rightarrow A \\
& \Gamma \vdash s: B \\
\text { 3. } & \Gamma \vdash t: C \Rightarrow(B \Rightarrow A) \\
& \Gamma \vdash r: C
\end{array}
$$


4. $C \Rightarrow(B \Rightarrow A) \equiv(C \wedge B) \Rightarrow A$

5.

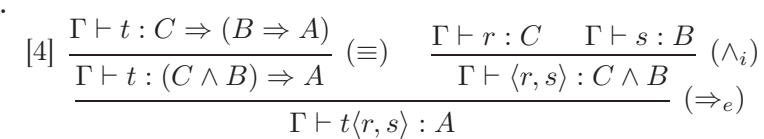

$\left(\right.$ P-сомм $\left._{\forall_{i} \rightarrow}\right): \Lambda X . \lambda x^{A} . t \rightleftarrows \lambda x^{A} . \Lambda X . t$

$(\rightarrow)$

1. $X \notin F T V(A)$

2. $\Gamma \vdash \Lambda X . \lambda x^{A} . t: B$

3. $B \equiv \forall X . C$

$\Gamma \vdash \lambda x^{A} . t: C$

$X \notin F T V(\Gamma)$

4. $C \equiv A \Rightarrow D$

$\Gamma, x: A \vdash t: D$

5. $\forall X .(A \Rightarrow D) \equiv A \Rightarrow \forall X . D$

6. $\forall X . C \equiv \forall X .(A \Rightarrow D)$

7.

$$
\begin{aligned}
& \text { [1 3] } \frac{\Gamma, x: A \vdash t: D}{\Gamma, x: A \vdash \Lambda X . t: \forall X . D}\left(\forall_{i}\right)
\end{aligned}
$$

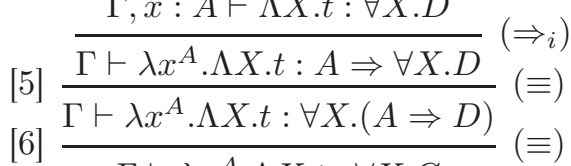

$$
\begin{aligned}
& \text { [6] } \frac{\Gamma] \frac{\Gamma \vdash \lambda x^{A} \cdot \Lambda X . t: \forall X . C}{\Gamma \vdash \lambda x^{A} . \Lambda X . t \cdot B}(\equiv)}{[\equiv)}
\end{aligned}
$$

$(\leftarrow)$

1. $X \notin F T V(A)$

(Hypothesis)

2. $\Gamma \vdash \lambda x^{A} . \Lambda X . t: B$

(Hypothesis)

3. $B \equiv A \Rightarrow C$

$\Gamma, x: A \vdash \Lambda X . t: C$

4. $C \equiv \forall X . D$

$\Gamma, x: A \vdash t: D$

$X \notin F T V(\Gamma) \cup F T V(A)$

5. $\forall X .(A \Rightarrow D) \equiv A \Rightarrow \forall X . D$

6. $A \Rightarrow C \equiv A \Rightarrow \forall X . D$

7.

$(\rightarrow)$

1. $X \notin F T V(A)$

(Hypothesis)

2. $\Gamma \vdash\left(\lambda x^{A} \cdot t\right)[B]: C$

(Hypothesis)

3. $C \equiv[X:=B] D$

$\Gamma \vdash \lambda x^{A} . t: \forall X . D$

(2, Lemma 4.12)

4. $\forall X . D \equiv A \Rightarrow E$

$\Gamma, x: A \vdash t: E$

(3, Lemma 4.12) 
5. $E \equiv \forall X . E^{\prime}$

$D \equiv A \Rightarrow E^{\prime}$

6. $A \Rightarrow[X:=B] E^{\prime}=[X:=B]\left(A \Rightarrow E^{\prime}\right)$

7. $[X:=B]\left(A \Rightarrow E^{\prime}\right) \equiv[X:=B] D$

8.

$$
\begin{aligned}
& {[5] \frac{\Gamma, x: A \vdash t: E}{\Gamma, x: A \vdash t: \forall X . E^{\prime}}(\equiv)} \\
& {[6] \frac{\frac{\frac{\Gamma, x: A \vdash t[B]:[X:=B] E^{\prime}}{\Gamma \vdash \lambda x^{A} \cdot t[B]: A \Rightarrow[X:=B] E^{\prime}}}{\Gamma \vdash \lambda x^{A} \cdot t[B]:[X:=B]\left(A \Rightarrow E^{\prime}\right)}}{[7]}\left({ }_{i}\right)}
\end{aligned}
$$

$(\leftarrow)$

1. $X \notin F T V(A)$

(Hypothesis)

2. $\Gamma \vdash \lambda x^{A} \cdot t[B]: C$

(Hypothesis)

3. $C \equiv A \Rightarrow D$

$\Gamma, x: A \vdash t[B]: D$

(1, Lemma 4.12)

4. $D \equiv[X:=B] E$

$\Gamma, x: A \vdash t: \forall X . E$

(2, Lemma 4.12)

5. $A \Rightarrow \forall X . E \equiv \forall X .(A \Rightarrow E)$

6. $[X:=B](A \Rightarrow E)=A \Rightarrow[X:=B] E$

(Iso. (5))

7. $A \Rightarrow[X:=B] E \equiv A \Rightarrow D$

8.

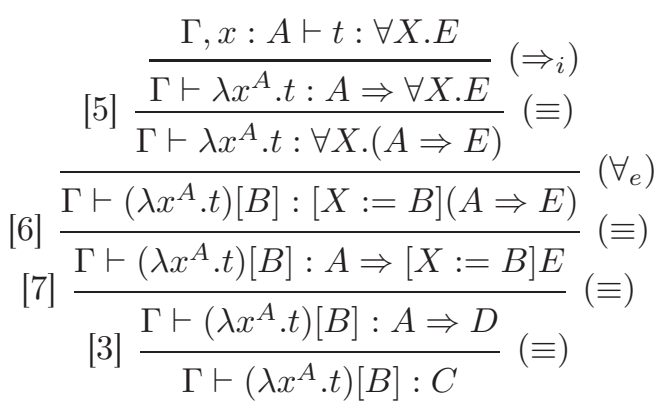

$\left(\mathrm{P}-\mathrm{DIST}_{\forall_{i} \wedge_{i}}\right): \Lambda X .\langle t, r\rangle \rightleftarrows\langle\Lambda X . t, \Lambda X . r\rangle$

$(\rightarrow)$

1. $\Gamma \vdash \Lambda X .\langle t, r\rangle: A$

(Hypothesis)

2. $A \equiv \forall X . B$

$\Gamma \vdash\langle t, r\rangle: B$

$X \notin F T V(\Gamma)$

(1, Lemma 4.12)

3. $B \equiv C \wedge D$

$\Gamma \vdash t: C$

$\Gamma \vdash r: D$

(2, Lemma 4.12)

(Iso. (6))

4. $\forall X .(C \wedge D) \equiv \forall X . C \wedge \forall X . D$

5. $\forall X . B \equiv \forall X .(C \wedge D)$

6.

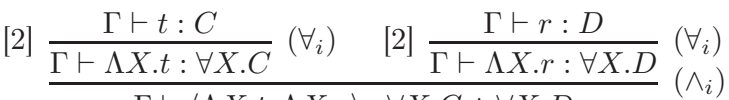

$$
\begin{aligned}
& \text { [4] } \frac{\Gamma \vdash\langle\Lambda X . t, \Lambda X . r\rangle: \forall X . C \wedge \forall X . D}{\Gamma \vdash\langle\Lambda X . t, \Lambda X . r\rangle: \forall X .(C \wedge D)}(\equiv) \\
& {[5] \frac{\Gamma \vdash\langle\Lambda X . t, \Lambda X . r\rangle: \forall X .(C \wedge D)}{\Gamma \vdash\langle\Lambda X . t, \Lambda X . r\rangle: \forall X . B}(\equiv)} \\
& {[2] \frac{\Gamma \vdash\langle\Lambda X . t, \Lambda X . r\rangle: \forall X . B}{\Gamma \vdash\langle\Lambda X . t, \Lambda X . r\rangle: A}(\equiv)}
\end{aligned}
$$


$(\leftarrow)$

1. $\Gamma \vdash\langle\Lambda X . t, \Lambda X . r\rangle: A$

(Hypothesis)

2. $A \equiv B \wedge C$

$\Gamma \vdash \Lambda X . t: B$

$\Gamma \vdash \Lambda X . r: C$

(1, Lemma 4.12)

3. $B \equiv \forall X . D$

$\Gamma \vdash t: D$

$X \notin F T V(\Gamma)$

(2, Lemma 4.12)

4. $C \equiv \forall X . E$

$\Gamma \vdash r: E$

$X \notin F T V(\Gamma)$

(2, Lemma 4.12)

5. $\forall X .(D \wedge E) \equiv \forall X . D \wedge \forall X . E$

6. $\forall X . D \wedge \forall X . E \equiv B \wedge C$

7.

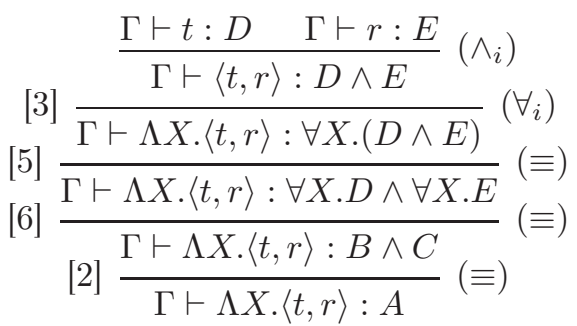

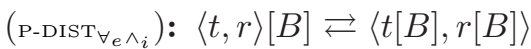

$(\rightarrow)$

1. $\Gamma \vdash\langle t, r\rangle[B]: A$

(Hypothesis)

2. $A \equiv[X:=B] C$

$\Gamma \vdash\langle t, r\rangle: \forall X . C$

(1, Lemma 4.12)

3. $\forall X . C \equiv D \wedge E$

$\Gamma \vdash t: D$

$\Gamma \vdash r: E$

(2, Lemma 4.12)

4. $D \equiv \forall X . D^{\prime}$

$E \equiv \forall X . E^{\prime}$

$C \equiv D^{\prime} \wedge E^{\prime}$

(3, Lemma 4.9)

5. $[X:=B]\left(D^{\prime} \wedge E^{\prime}\right)=[X:=B] D^{\prime} \wedge[X:=B] E^{\prime}$

(4, congr. ( $\equiv))$

6. $[X:=B] C \equiv[X:=B]\left(D^{\prime} \wedge E^{\prime}\right)$

7.

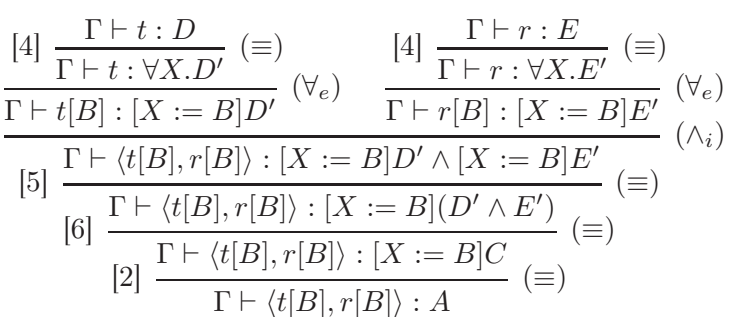

$(\leftarrow)$

1. $\Gamma \vdash\langle t[B], r[B]\rangle: A$

(Hypothesis)

2. $A \equiv C \wedge D$

$\Gamma \vdash t[B]: C$

$\Gamma \vdash r[B]: D$

(1, Lemma 4.12)

3. $C \equiv[X:=B] C^{\prime}$

$\Gamma \vdash t: \forall X . C^{\prime}$

(2, Lemma 4.12)

4. $D \equiv[X:=B] D^{\prime}$

$\Gamma \vdash r: \forall X . D^{\prime}$

(2, Lemma 4.12) 
5. $\forall X .\left(C^{\prime} \wedge D^{\prime}\right) \equiv \forall X . C^{\prime} \wedge \forall X . D^{\prime}$

6. $[X:=B]\left(C^{\prime} \wedge D^{\prime}\right)=[X:=B] C^{\prime} \wedge[X:=B] D^{\prime}$

(Def.)

7. $[X:=B] C^{\prime} \wedge[X:=B] D^{\prime} \equiv C \wedge D$

$(3,4$, congr. $(\equiv))$

8.

$$
\begin{aligned}
& \frac{\Gamma \vdash t: \forall X . C^{\prime} \quad \Gamma \vdash r: \forall Y . D^{\prime}}{\Gamma \vdash\langle t, r\rangle: \forall X . C^{\prime} \wedge \forall X . D^{\prime}}\left(\wedge_{i}\right) \\
& \text { [5] } \frac{\Gamma \vdash\langle t, r\rangle: \forall X . C^{\prime} \wedge \forall X . D^{\prime}}{\Gamma \vdash\langle t, r\rangle: \forall X .\left(C^{\prime} \wedge D^{\prime}\right)}(\equiv)
\end{aligned}
$$

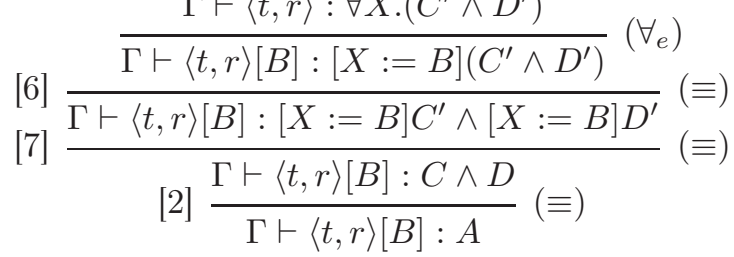

$\left({\mathrm{P}-\mathrm{DIST}_{\forall_{i} \wedge e}}\right): \pi_{\forall X . B}(\Lambda X . t) \rightleftarrows \Lambda X . \pi_{B}(t)$

$(\rightarrow)$

1. $\Gamma \vdash \pi_{\forall X . B}(\Lambda X . t): A$

(Hypothesis)

2. $A \equiv \forall X . B$

$\Gamma \vdash \Lambda X . t:(\forall X . B) \wedge C$

(1, Lemma 4.12)

3. $(\forall X . B) \wedge C \equiv \forall X . D$

$\Gamma \vdash t: D$

$X \notin F T V(\Gamma)$

(2, Lemma 4.12)

4. $C \equiv \forall X . C^{\prime}$

$D \equiv B \wedge C^{\prime}$

(3, Lemma 4.9)

5.

$$
\begin{gathered}
{[4] \frac{\Gamma \vdash t: D}{\frac{\Gamma \vdash t: B \wedge C^{\prime}}{\Gamma \vdash \pi_{B}(t): B}\left({ }^{\prime}\right)}} \\
{[3] \frac{\left(_{e}\right)}{\Gamma \vdash \Lambda X . \pi_{B}(t): \forall X . B}\left(\forall_{i}\right)} \\
[2])
\end{gathered}
$$

$(\leftarrow)$

1. $\Gamma \vdash \Lambda X . \pi_{B}(t): A$

2. $A \equiv \forall X . C$

$\Gamma \vdash \pi_{B}(t): C$

$X \notin F T V(\Gamma)$

(1, Lemma 4.12)

3. $B \equiv C$

$\Gamma \vdash t: C \wedge D$

(2, Lemma 4.12)

4. $\forall X .(C \wedge D) \equiv \forall X . C \wedge \forall X . D$

(Iso. (6))

5 .

$$
\begin{aligned}
& {[2] \frac{\Gamma \vdash t: C \wedge D}{\Gamma \vdash \Lambda X . t: \forall X .(C \wedge D)}\left({ }_{i}\right)} \\
& {[4] \frac{}{\Gamma \vdash \Lambda X . t: \forall X . C \wedge \forall X . D}} \\
& {\left[\begin{array}{l}
\Gamma \vdash \pi_{\forall X . B}(\Lambda X . t): \forall X . C \\
\Gamma \vdash \pi_{\forall X . B}(\Lambda X . t): A
\end{array}\left({ }_{e}\right)\right.}
\end{aligned}
$$

$\left({\mathrm{P}-\mathrm{DIST}_{\forall e} \wedge e}\right):\left(\pi_{\forall X . B}(t)\right)[C] \rightleftarrows \pi_{[X:=C] B}(t[C])$

$(\rightarrow)$

1. $\Gamma \vdash t: \forall X .(B \wedge D)$

(Hypothesis)

2. $\Gamma \vdash\left(\pi_{\forall X . B}(t)\right)[C]: A$

(Hypothesis)

3. $A \equiv[X:=C] E$

$\Gamma \vdash \pi_{\forall X . B}(t): \forall X . E$

(2, Lemma 4.12) 
4. $\forall X . E \equiv \forall X . B$ $\Gamma \vdash t: \forall X . E \wedge F$

5. $E \equiv B$

(3, Lemma 4.12)

6. $[X:=C](B \wedge D)=[X:=C] B \wedge[X:=C] D$

7. $[X:=C] B \equiv[X:=C] E$

8.

$$
\begin{gathered}
{[6] \frac{\frac{\Gamma \vdash t: \forall X . B \wedge D}{\Gamma \vdash t[C]:[X:=C](B \wedge D)}}{\frac{\Gamma \vdash t[C]:[X:=C] B \wedge[X:=C] D}{\Gamma \vdash \pi_{[X:=C] B}(t[C]):[X:=C] B}}\left(\forall_{e}\right)} \\
{[7] \frac{\left(\wedge_{e}\right)}{\Gamma \vdash \pi_{[X:=C] B}(t[C]):[X:=C] E}} \\
{[3] \frac{(\equiv)}{\Gamma \vdash \pi_{[X:=C] B}(t[C]): A}(\equiv)}
\end{gathered}
$$

$(\leftarrow)$

1. $\Gamma \vdash t: \forall X .(B \wedge D)$

(Hypothesis)

2. $\Gamma \vdash \pi_{[X:=C] B}(t[C]): A$

3. $A \equiv[X:=C] B$

$\Gamma \vdash t[C]: A \wedge E$

4. $\forall X .(B \wedge D) \equiv \forall X . B \wedge \forall X . D$

(5, congr. $(\equiv))$

5 .

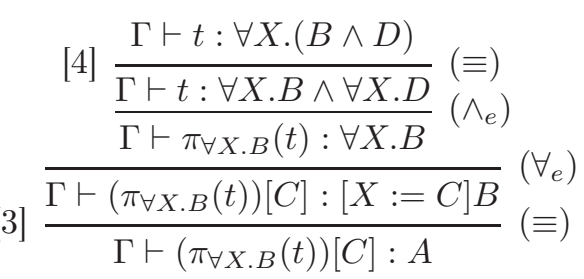

$\left(\beta_{\lambda}\right):$ If $\Gamma \vdash s: A,\left(\lambda x^{A} \cdot r\right) s \hookrightarrow[x:=s] r$

1. $\Gamma \vdash s: A$

(Hypothesis)

2. $\Gamma \vdash \lambda x^{A} . r: B$

(Hypothesis)

3. $\Gamma \vdash \lambda x^{A} . r: A \Rightarrow B$

(2, Lemma 4.12)

4. $A \Rightarrow B \equiv A \Rightarrow C$

$\Gamma, x: A \vdash r: C$

(3, Lemma 4.12)

5. $B \equiv C$

(4, congr. ( $\equiv))$

6. $\Gamma \vdash[x:=s] r: C$

(1, 4, Lemma 4.13)

7. $\Gamma \vdash[x:=s] r: B$

$(5,6$, rule $(\equiv))$

$\left(\beta_{\Lambda}\right):(\Lambda X . r)[A] \hookrightarrow[X:=A] r$

1. $\Gamma \vdash(\Lambda X . r)[A]: B$

(Hypothesis)

2. $B \equiv[X:=A] C$

$\Gamma \vdash \Lambda X . r: \forall X . C$

(1, Lemma 4.12)

3. $\forall X . C \equiv \forall X . D$

$\Gamma \vdash r: D$

$X \notin F T V(\Gamma)$

(2, Lemma 4.12)

4. $C \equiv D$

5. $\Gamma \vdash r: C$

$(4$, rule $(\equiv))$

6. $[X:=A] \Gamma \vdash \Gamma \vdash[X:=A] r:[X:=A] C$

(5, Lemma 4.13) 
$(\pi)$ If $\Gamma \vdash r: A, \pi_{A}(\langle r, s\rangle) \hookrightarrow r$

1. $\Gamma \vdash r: A$

(Hypothesis)

2. $\pi_{A}(\langle r, s\rangle): B$

(Hypothesis)

3. $B \equiv A$

$\Gamma \vdash\langle r, s\rangle: A \wedge C$

(2, Lemma 4.12)

4. $\Gamma \vdash \pi_{A}(\langle r, s\rangle): A$

$(2,3$, rule $(\equiv))$

\section{B Detailed proof of Section 5.3}

Lemma 5.18. For all $r, s, t$ such that $\langle r, s\rangle \rightleftarrows * t$, we have either

1. $t=\langle u, v\rangle$ where either

(a) $u \rightleftarrows^{*}\left\langle t_{11}, t_{21}\right\rangle$ and $v \rightleftarrows^{*}\left\langle t_{12}, t_{22}\right\rangle$ with $r \rightleftarrows^{*}\left\langle t_{11}, t_{12}\right\rangle$ and $s \rightleftarrows^{*}\left\langle t_{21}, t_{22}\right\rangle$, or

(b) $v \rightleftarrows^{*}\langle w, s\rangle$ with $r \rightleftarrows^{*}\langle u, w\rangle$, or any of the three symmetric cases, or

(c) $r \rightleftarrows^{*} u$ and $s \rightleftarrows^{*} v$, or the symmetric case.

2. $t=\lambda x^{A}$. $a$ and $a \rightleftarrows^{*}\left\langle a_{1}, a_{2}\right\rangle$ with $r \rightleftarrows^{*} \lambda x^{A} . a_{1}$ and $s \rightleftarrows^{*} \lambda x^{A} . a_{2}$.

3. $t=a v$ and $a \rightleftarrows^{*}\left\langle a_{1}, a_{2}\right\rangle$, with $r \rightleftarrows^{*} a_{1} v$ and $s \rightleftarrows^{*} a_{2} v$.

4. $t=\Lambda X$. a and $a \rightleftarrows^{*}\left\langle a_{1}, a_{2}\right\rangle$ with $r \rightleftarrows^{*} \Lambda X . a_{1}$ and $s \rightleftarrows^{*} \Lambda X . a_{2}$.

5. $t=a[A]$ and $a \rightleftarrows^{*}\left\langle a_{1}, a_{2}\right\rangle$, with $r \rightleftarrows^{*} a_{1}[A]$ and $s \rightleftarrows^{*} a_{2}[A]$.

Proof. By a double induction, first on $M(t)$ and then on the length of the relation $\rightleftarrows *$. Consider an equivalence proof $\langle r, s\rangle \rightleftarrows^{*} t^{\prime} \rightleftarrows t$ with a shorter proof $\langle r, s\rangle \rightleftarrows^{*} t^{\prime}$. By the second induction hypothesis, the term $t^{\prime}$ has the form prescribed by the lemma. We consider the five cases and in each case, the possible rules transforming $t^{\prime}$ in $t$.

1. Let $\langle r, s\rangle \rightleftarrows^{*}\langle u, v\rangle \rightleftarrows t$. The possible equivalences from $\langle u, v\rangle$ are

- $t=\left\langle u^{\prime}, v\right\rangle$ or $\left\langle u, v^{\prime}\right\rangle$ with $u \rightleftarrows u^{\prime}$ and $v \rightleftarrows v^{\prime}$, and so the term $t$ is in case 1 .

- Rules (сомм) and (Asso) preserve the conditions of case 1.

- $t=\lambda x^{A} \cdot\left\langle u^{\prime}, v^{\prime}\right\rangle$, with $u=\lambda x^{A} \cdot u^{\prime}$ and $v=\lambda x^{A} \cdot v^{\prime}$, and so the term $t$ is in case 2 .

- $t=\left\langle u^{\prime}, v^{\prime}\right\rangle t^{\prime}$, with $u=u^{\prime} t^{\prime}$ and $v=v^{\prime} t^{\prime}$, and so the term $t$ is in case 3 .

- $t=\Lambda X .\left\langle u^{\prime}, v^{\prime}\right\rangle$, with $u=\Lambda X . u^{\prime}$ and $v=\Lambda X . v^{\prime}$, and so the term $t$ is in case 4 .

- $t=\left\langle u^{\prime}, v^{\prime}\right\rangle[A]$, with $u=u^{\prime}[A]$ and $v=v^{\prime}[A]$, and so the term $t$ is in case 5 .

2. Let $\langle r, s\rangle \rightleftarrows^{*} \lambda x^{A} . a \rightleftarrows t$, with $a \rightleftarrows^{*}\left\langle a_{1}, a_{2}\right\rangle, r \rightleftarrows^{*} \lambda x^{A} . a_{1}$, and $s \rightleftarrows^{*} \lambda x^{A} \cdot a_{2}$. Hence, possible equivalences from $\lambda x^{A} . a$ to $t$ are

- $t=\lambda x^{A} \cdot a^{\prime}$ with $a \rightleftarrows^{*} a^{\prime}$, hence $a^{\prime} \rightleftarrows\left\langle a_{1}, a_{2}\right\rangle$, and so the term $t$ is in case 2 .

- $t=\left\langle\lambda x^{A} \cdot u, \lambda x^{A} \cdot v\right\rangle$, with $\left\langle a_{1}, a_{2}\right\rangle \rightleftarrows^{*} a=\langle u, v\rangle$. Hence, by the first induction hypothesis (since $M(a)<M(t)$ ), either

(a) $a_{1} \rightleftarrows^{*} u$ and $a_{2} \rightleftarrows^{*} v$, and so $r \rightleftarrows^{*} \lambda x^{A} . u$ and $s \rightleftarrows^{*} \lambda x^{A} . v$, or

(b) $v \rightleftarrows^{*}\left\langle t_{1}, t_{2}\right\rangle$ with $a_{1} \rightleftarrows^{*}\left\langle u, t_{1}\right\rangle$ and $a_{2} \rightleftarrows^{*} t_{2}$, and so $\lambda x^{A} . v \rightleftarrows^{*}\left\langle\lambda x^{A} \cdot t_{1}, \lambda x^{A} \cdot t_{2}\right\rangle$, $r \rightleftarrows^{*}\left\langle\lambda x^{A} . u, \lambda x^{A} . t_{1}\right\rangle$ and $s \rightleftarrows * x^{A} . t_{2}$, or 
(c) $u \rightleftarrows^{*}\left\langle t_{11}, t_{21}\right\rangle$ and $v \rightleftarrows^{*}\left\langle t_{12}, t_{22}\right\rangle$ with $a_{1} \rightleftarrows^{*}\left\langle t_{11}, t_{12}\right\rangle$ and $a_{2} \rightleftarrows^{*}\left\langle t_{21}, t_{22}\right\rangle$, and so $\lambda x^{A} . u \rightleftarrows^{*}\left\langle\lambda x^{A} . t_{11}, \lambda x^{A} . t_{21}\right\rangle, \lambda x^{A} . v \rightleftarrows^{*}\left\langle\lambda x^{A} . t_{12}, \lambda x^{A} . t_{22}\right\rangle, r \rightleftarrows^{*}\left\langle\lambda x^{A} . t_{11}, \lambda x^{A} . t_{12}\right\rangle$ and $s \rightleftarrows^{*}\left\langle\lambda x^{A} \cdot t_{21}, \lambda x^{A} \cdot t_{22}\right\rangle$.

(the symmetric cases are analogous), and so the term $t$ is in case 1 .

- $t=\Lambda X . \lambda x^{A} \cdot a^{\prime}$ with $a=\Lambda X . a^{\prime}$, hence $\Lambda X . a^{\prime} \rightleftarrows^{*}\left\langle a_{1}, a_{2}\right\rangle$. Since $M\left(\left\langle a_{1}, a_{2}\right\rangle\right)<M(\langle r, s\rangle)$, by the first induction hypothesis, the term $t$ is in case 4 .

- $t=\lambda x^{A} \cdot a^{\prime}[B]$ with $a=a^{\prime}[B]$, hence $a^{\prime}[B] \rightleftarrows^{*}\left\langle a_{1}, a_{2}\right\rangle$. Since $M\left(\left\langle a_{1}, a_{2}\right\rangle\right)<M(\langle r, s\rangle)$, by the first induction hypothesis, the term $t$ is in case 5 .

3. Let $\langle r, s\rangle \rightleftarrows^{*} a w \rightleftarrows t$, with $a \rightleftarrows^{*}\left\langle a_{1}, a_{2}\right\rangle, r \rightleftarrows^{*} a_{1} w$, and $s \rightleftarrows^{*} a_{2} w$. The possible equivalences from aw to $t$ are

- $t=a^{\prime} w$ with $a \rightleftarrows^{*} a^{\prime}$, hence $a^{\prime} \rightleftarrows^{*}\left\langle a_{1}, a_{2}\right\rangle$, and so the term $t$ is in case 3 .

- $t=a w^{\prime}$ with $w \rightleftarrows^{*} w^{\prime}$ and so the term $t$ is in case 3 .

- $t=\langle u w, v w\rangle$, with $\left\langle a_{1}, a_{2}\right\rangle a_{2} \rightleftarrows^{*} a=\langle u, v\rangle$. Hence, by the first induction hypothesis (since $M(a)<M(t))$, either

(a) $a_{1} \rightleftarrows^{*} u$ and $a_{2} \rightleftarrows^{*} v$, and so $r \rightleftarrows^{*} u w$ and $s \rightleftarrows^{*} v w$, or

(b) $v \rightleftarrows^{*}\left\langle t_{1}, t_{2}\right\rangle$ with $a_{1} \rightleftarrows^{*}\left\langle u, t_{1}\right\rangle$ and $a_{2} \rightleftarrows^{*} t_{2}$, and so $v w \rightleftarrows^{*}\left\langle t_{1} w, t_{2} w\right\rangle, r \rightleftarrows^{*}\left\langle u w, t_{1} w\right\rangle$ and $s \rightleftarrows^{*} t_{2} w$, or

(c) $u \rightleftarrows^{*}\left\langle t_{11}, t_{21}\right\rangle$ and $v \rightleftarrows^{*}\left\langle t_{12}, t_{22}\right\rangle$ with $a_{1} \rightleftarrows^{*}\left\langle t_{11}, t_{12}\right\rangle$ and $a_{2} \rightleftarrows^{*}\left\langle t_{21}, t_{22}\right\rangle$, and so $u w \rightleftarrows^{*}\left\langle t_{11} w, t_{21} w\right\rangle, v w \rightleftarrows^{*}\left\langle t_{12} w, t_{22} w\right\rangle, r \rightleftarrows^{*}\left\langle t_{11} w, t_{12} w\right\rangle$ and $s \rightleftarrows^{*}\left\langle t_{21} w, t_{22} w\right\rangle$.

(the symmetric cases are analogous), and so the term $t$ is in case 1 .

- $t=a^{\prime}\langle v, w\rangle$ with $a=a^{\prime} v$, thus $a^{\prime} v=a \rightleftarrows^{*}\left\langle a_{1}, a_{2}\right\rangle$. Hence, by the first induction hypothesis, $a^{\prime} \rightleftarrows^{*}\left\langle a_{1}^{\prime}, a_{2}^{\prime}\right\rangle$, with $a_{1} \rightleftarrows^{*} a_{1}^{\prime} v$ and $a_{2} \rightleftarrows^{*} a_{2}^{\prime} v$. Therefore, $r \rightleftarrows^{*} a_{1}^{\prime}\langle v, w\rangle$ and $s \rightleftarrows^{*} a_{2}^{\prime}\langle v, w\rangle$, and so the term $t$ is in case 3 .

4. Let $\langle r, s\rangle \rightleftarrows^{*} \Lambda X . a \rightleftarrows t$, with $a \rightleftarrows^{*}\left\langle a_{1}, a_{2}\right\rangle, r \rightleftarrows^{*} \Lambda X . a_{1}$, and $s \rightleftarrows^{*} \Lambda X . a_{2}$. Hence, possible equivalences from $\lambda x . a$ to $t$ are

- $t=\Lambda X$. $a^{\prime}$ with $a \rightleftarrows^{*} a^{\prime}$, hence $a^{\prime} \rightleftarrows\left\langle a_{1}, a_{2}\right\rangle$, and so the term $t$ is in case 4 .

- $t=\langle\Lambda X . u, \Lambda X . v\rangle$, with $\left\langle a_{1}, a_{2}\right\rangle \rightleftarrows^{*} a=\langle u, v\rangle$. Hence, by the first induction hypothesis (since $M(a)<M(t))$, either

(a) $a_{1} \rightleftarrows^{*} u$ and $a_{2} \rightleftarrows^{*} v$, and so $r \rightleftarrows^{*} \Lambda X$.u and $s \rightleftarrows^{*} \Lambda X$.v, or

(b) $v \rightleftarrows^{*}\left\langle t_{1}, t_{2}\right\rangle$ with $a_{1} \rightleftarrows^{*}\left\langle u, t_{1}\right\rangle$ and $a_{2} \rightleftarrows^{*} t_{2}$, and so $\Lambda X . v \rightleftarrows^{*}\left\langle\Lambda X . t_{1}, \Lambda X . t_{2}\right\rangle, r \rightleftarrows^{*}$ $\left\langle\Lambda X . u, \Lambda X . t_{1}\right\rangle$ and $s \rightleftarrows^{*} \Lambda X . t_{2}$, or

(c) $u \rightleftarrows^{*}\left\langle t_{11}, t_{21}\right\rangle$ and $v \rightleftarrows^{*}\left\langle t_{12}, t_{22}\right\rangle$ with $a_{1} \rightleftarrows^{*}\left\langle t_{11}, t_{12}\right\rangle$ and $a_{2} \rightleftarrows^{*}\left\langle t_{21}, t_{22}\right\rangle$, and so $\Lambda X . u \rightleftarrows^{*}\left\langle\Lambda X . t_{11}, \Lambda X . t_{21}\right\rangle, \Lambda X . v \rightleftarrows^{*}\left\langle\Lambda X . t_{12}, \Lambda X . t_{22}\right\rangle, r \rightleftarrows^{*}\left\langle\Lambda X . t_{11}, \Lambda X . t_{12}\right\rangle$ and $s \rightleftarrows^{*}$ $\left\langle\Lambda X . t_{21}, \Lambda X . t_{22}\right\rangle$.

(the symmetric cases are analogous), and so the term $t$ is in case 1 .

- $t=\lambda x^{A} . \Lambda X$. $a^{\prime}$ with $a=\lambda x^{A} \cdot a^{\prime}$, hence $\lambda x^{A} \cdot a^{\prime} \rightleftarrows^{*}\left\langle a_{1}, a_{2}\right\rangle$. Since $M\left(\left\langle a_{1}, a_{2}\right\rangle\right)<M(\langle r, s\rangle)$, by the first induction hypothesis, the term $t$ is in case 2 .

- $t=\left(\Lambda X . a^{\prime}\right)[B]$ with $a=a^{\prime}[B]$, hence $a^{\prime}[B] \rightleftarrows^{*}\left\langle a_{1}, a_{2}\right\rangle$. Since $M\left(\left\langle a_{1}, a_{2}\right\rangle\right)<M(\langle r, s\rangle)$, by the first induction hypothesis, the term $t$ is in case 5 .

5. Let $\langle r, s\rangle \rightleftarrows^{*} a[A] \rightleftarrows t$, with $a \rightleftarrows^{*}\left\langle a_{1}, a_{2}\right\rangle, r \rightleftarrows^{*} a_{1}[A]$, and $s \rightleftarrows^{*} a_{2}[A]$. The possible equivalences from $a[A]$ to $t$ are

- $t=a^{\prime}[A]$ with $a \rightleftarrows^{*} a^{\prime}$, hence $a^{\prime} \rightleftarrows^{*}\left\langle a_{1}, a_{2}\right\rangle$, and so the term $t$ is in case 5 .

- $t=\langle u[A], v[A]\rangle$, with $\left\langle a_{1}, a_{2}\right\rangle \rightleftarrows^{*} a=\langle u, v\rangle$. Hence, by the first induction hypothesis (since $M(a)<M(t))$, either

(a) $a_{1} \rightleftarrows^{*} u$ and $a_{2} \rightleftarrows^{*} v$, and so $r \rightleftarrows^{*} u[A]$ and $s \rightleftarrows^{*} v[A]$, or

(b) $v \rightleftarrows^{*}\left\langle t_{1}, t_{2}\right\rangle$ with $a_{1} \rightleftarrows^{*}\left\langle u, t_{1}\right\rangle$ and $a_{2} \rightleftarrows^{*} t_{2}$, and so $v[A] \rightleftarrows^{*}\left\langle t_{1}[A], t_{2}[A]\right\rangle, r \rightleftarrows^{*}$ $\left\langle u[A], t_{1}[A]\right\rangle$ and $s \rightleftarrows^{*} t_{2}[A]$, or 
(c) $u \rightleftarrows^{*}\left\langle t_{11}, t_{21}\right\rangle$ and $v \rightleftarrows^{*}\left\langle t_{12}, t_{22}\right\rangle$ with $a_{1} \rightleftarrows^{*}\left\langle t_{11}, t_{12}\right\rangle$ and $a_{2} \rightleftarrows^{*}\left\langle t_{21}, t_{22}\right\rangle$, and so $u[A] \rightleftarrows^{*}\left\langle t_{11}[A], t_{21}[A]\right\rangle, v[A] \rightleftarrows^{*}\left\langle t_{12}[A], t_{22}[A]\right\rangle, r \rightleftarrows^{*}\left\langle t_{11}[A], t_{12}[A]\right\rangle$ and $s \rightleftarrows^{*}$ $\left\langle t_{21}[A], t_{22}[A]\right\rangle$.

(the symmetric cases are analogous), and so the term $t$ is in case 1 .

- $t=\lambda x^{B} \cdot\left(a^{\prime}[A]\right)$ with $a=\lambda x^{B} \cdot a^{\prime}$, hence $\lambda x^{B} \cdot a^{\prime} \rightleftarrows^{*}\left\langle a_{1}, a_{2}\right\rangle$. Since $M\left(\lambda x^{B} \cdot a^{\prime}\right)<M(\langle r, s\rangle)$, by the first induction hypothesis, the term $t$ is in case 2 .

- $t=\pi_{[X:=A] B}\left(a^{\prime}[A]\right)$ with $a=\pi_{\forall X . B}\left(a^{\prime}\right)$, hence $\pi_{\forall X . B}\left(a^{\prime}\right) \rightleftarrows *\left\langle a_{1}, a_{2}\right\rangle$. Since $M\left(\pi_{\forall X . B}\left(a^{\prime}\right)\right)<$ $M(\langle r, s\rangle)$, by the first induction hypothesis, this case is absurd. Indeed, $\left\langle a_{1}, a_{2}\right\rangle$ is never equivalent to $\pi_{\forall X . B}\left(a^{\prime}\right)$.

Lemma 5.19. For all $r_{1}, r_{2}, s, t$ such that $\left\langle r_{1}, r_{2}\right\rangle \rightleftarrows^{*} s \hookrightarrow t$, there exists $u_{1}, u_{2}$ such that $t \rightleftarrows^{*}\left\langle u_{1}, u_{2}\right\rangle$ and either

1. $r_{1} \rightarrow u_{1}$ and $r_{2} \rightarrow u_{2}$

2. $r_{1} \rightarrow u_{1}$ and $r_{2} \rightleftarrows^{*} u_{2}$, or

3. $r_{1} \rightleftarrows^{*} u_{1}$ and $r_{2} \rightarrow u_{2}$.

Proof. By induction on $M\left(\left\langle r_{1}, r_{2}\right\rangle\right)$. By Lemma 5.18, $s$ is either a product, an abstraction, an application, a type abstraction or a type application with the conditions given in the lemma. The different terms $s$ reducible by $\hookrightarrow$ are

- $\left(\lambda x^{A} . a\right) s^{\prime}$ that reduces by the $\left(\beta_{\lambda}\right)$ rule to $\left[x:=s^{\prime}\right] a$.

- $(\Lambda X . a)[A]$ that reduces by the $\left(\beta_{\Lambda}\right)$ rule to $[X:=A] a$.

- $\left\langle s_{1}, s_{2}\right\rangle, \lambda x^{A} . a, a s^{\prime}, \Lambda X . a, a[A]$ with a reduction in the subterm $s_{1}, s_{2}, a$, or $s^{\prime}$.

Notice that rule $(\pi)$ cannot apply since $s \not \not^{*} \pi_{C}\left(s^{\prime}\right)$.

We consider each case:

- $s=\left(\lambda x^{A} . a\right) s^{\prime}$ and $t=\left[x:=s^{\prime}\right] a$. Using twice Lemma 5.18, we have $a \rightleftarrows *\left\langle a_{1}, a_{2}\right\rangle, r_{1} \rightleftarrows^{*}\left(\lambda x^{A} . a_{1}\right) s^{\prime}$ and $r_{2} \rightleftarrows^{*}\left(\lambda x^{A} . a_{2}\right) s^{\prime}$. Since $t \rightleftarrows^{*}\left\langle\left[x:=s^{\prime}\right] a_{1},\left[x:=s^{\prime}\right] a_{2}\right\rangle$, we take $u_{1}=\left[x:=s^{\prime}\right] a_{1}$ and $u_{2}=\left[x:=s^{\prime}\right] a_{2}$.

- $s=(\Lambda X . a)[A]$ and $t=[X:=A] a$. Using twice Lemma 5.18, we have $a \rightleftarrows^{*}\left\langle a_{1}, a_{2}\right\rangle, r_{1} \rightleftarrows^{*}$ $\left(\Lambda X . a_{1}\right)[A]$ and $r_{2} \rightleftarrows^{*}\left(\Lambda X . a_{2}\right)[A]$. Since $t \rightleftarrows^{*}\left\langle[X:=A] a_{1},[X:=A] a_{2}\right\rangle$, we take $u_{1}=[X:=A] a_{1}$ and $u_{2}=[X:=A] a_{2}$.

- $s=\left\langle s_{1}, s_{2}\right\rangle, t=\left\langle t_{1}, s_{2}\right\rangle$ or $t=\left\langle s_{1}, t_{2}\right\rangle$, with $s_{1} \hookrightarrow t_{1}$ and $s_{2} \hookrightarrow t_{2}$. We only consider the first case since the other is analogous. One of the following cases happen

(a) $r_{1} \rightleftarrows^{*}\left\langle w_{11}, w_{21}\right\rangle, r_{2} \rightleftarrows^{*}\left\langle w_{12}, w_{22}\right\rangle, s_{1}=\left\langle w_{11}, w_{12}\right\rangle$ and $s_{2}=\left\langle w_{21}, w_{22}\right\rangle$. Hence, by the induction hypothesis, either $t_{1}=\left\langle w_{11}^{\prime}, w_{12}\right\rangle$, or $t_{1}=\left\langle w_{11}, w_{12}^{\prime}\right\rangle$, or $t_{1}=\left\langle w_{11}^{\prime}, w_{12}^{\prime}\right\rangle$, with $w_{11} \hookrightarrow w_{11}^{\prime}$ and $w_{12} \hookrightarrow w_{12}^{\prime}$. We take, in the first case $u_{1}=\left\langle w_{11}^{\prime}, w_{21}\right\rangle$ and $u_{2}=\left\langle w_{12}, w_{22}\right\rangle$, in the second case $u_{1}=\left\langle w_{11}, w_{21}\right\rangle$ and $u_{2}=\left\langle w_{12}^{\prime}, w_{22}\right\rangle$, and in the third $u_{1}=\left\langle w_{11}^{\prime}, w_{21}\right\rangle$ and $u_{2}=\left\langle w_{12}^{\prime}, w_{22}\right\rangle$.

(b) We consider two cases, since the other two are symmetric.

$-r_{1} \rightleftarrows^{*}\left\langle s_{1}, w\right\rangle$ and $s_{2} \rightleftarrows^{*}\left\langle w, r_{2}\right\rangle$, in which case we take $u_{1}=\left\langle t_{1}, w\right\rangle$ and $u_{2}=r_{2}$.

$-r_{2} \rightleftarrows^{*}\left\langle w, s_{2}\right\rangle$ and $s_{1}=\left\langle r_{1}, w\right\rangle$. Hence, by the induction hypothesis, either $t_{1}=\left\langle r_{1}^{\prime}, w\right\rangle$, or $t_{1}=\left\langle r_{1}, w^{\prime}\right\rangle$ or $t_{1}=\left\langle r_{1}^{\prime}, w^{\prime}\right\rangle$, with $r_{1} \hookrightarrow r_{1}^{\prime}$ and $w \hookrightarrow w^{\prime}$. We take, in the first case $u_{1}=r_{1}^{\prime}$ and $u_{2}=\left\langle w, s_{2}\right\rangle$, in the second case $u_{1}=r_{1}$ and $u_{2}=\left\langle w^{\prime}, s_{2}\right\rangle$, and in the third case $u_{1}=r_{1}^{\prime}$ and $u_{2}=\left\langle w^{\prime}, s_{2}\right\rangle$.

(c) $r_{1} \rightleftarrows^{*} s_{1}$ and $r_{2} \rightleftarrows^{*} s_{2}$, in which case we take $u_{1}=t_{1}$ and $u_{2}=s_{2}$.

- $s=\lambda x^{A}$. $a, t=\lambda x^{A} \cdot t^{\prime}$, and $a \hookrightarrow t^{\prime}$, with $a \rightleftarrows^{*}\left\langle a_{1}, a_{2}\right\rangle$ and $s \rightleftarrows^{*} \lambda x^{A} \cdot\left\langle a_{1}, a_{2}\right\rangle x^{A}$. $a_{2}$. Therefore, by the induction hypothesis, there exists $u_{1}^{\prime}, u_{2}^{\prime}$ such that either $\left(a_{1} \rightarrow u_{1}^{\prime}\right.$ and $\left.a_{2} \rightarrow u_{2}^{\prime}\right)$, or $\left(a_{1} \rightleftarrows^{*} u_{1}^{\prime}\right.$ and $\left.a_{2} \rightarrow u_{2}^{\prime}\right)$, or $\left(a_{1} \rightarrow u_{1}^{\prime}\right.$ and $\left.a_{2} \rightleftarrows^{*} u_{2}^{\prime}\right)$. Therefore, we take $u_{1}=\lambda x^{A} \cdot u_{1}^{\prime}$ and $u_{2}=\lambda x^{A} \cdot u_{2}^{\prime}$. 
- $s=a s^{\prime}, t=t^{\prime} s^{\prime}$, and $a \hookrightarrow t^{\prime}$, with $a \rightleftarrows^{*}\left\langle a_{1}, a_{2}\right\rangle$ and $s \rightleftarrows^{*}\left\langle a_{1} s^{\prime}, a_{2} s^{\prime}\right\rangle$. Therefore, by the induction hypothesis, there exists $u_{1}^{\prime}, u_{2}^{\prime}$ such that either $\left(a_{1} \rightarrow u_{1}^{\prime}\right.$ and $\left.a_{2} \rightarrow u_{2}^{\prime}\right)$, or $\left(a_{1} \rightleftarrows^{*} u_{1}^{\prime}\right.$ and $\left.a_{2} \rightarrow u_{2}^{\prime}\right)$, or $\left(a_{1} \rightarrow u_{1}^{\prime}\right.$ and $\left.a_{2} \rightleftarrows^{*} u_{2}^{\prime}\right)$. Therefore, we take $u_{1}=u_{1}^{\prime} s^{\prime}$ and $u_{2}=u_{2}^{\prime} s^{\prime}$.

- $s=a s^{\prime}, t=a t^{\prime}$, and $s^{\prime} \hookrightarrow t^{\prime}$, with $a \rightleftarrows^{*}\left\langle a_{1}, a_{2}\right\rangle$ and $s \rightleftarrows^{*}\left\langle a_{1} s^{\prime}, a_{2} s^{\prime}\right\rangle$. By Lemma 5.18 several times, one the following cases happen

(a) $a_{1} s^{\prime} \rightleftarrows^{*}\left\langle w_{11} s^{\prime}, w_{12} s^{\prime}\right\rangle, a_{2} s^{\prime} \rightleftarrows^{*}\left\langle w_{21} s^{\prime}, w_{22} s^{\prime}\right\rangle, r_{1} \rightleftarrows^{*}\left\langle w_{11} s^{\prime}, w_{21} s^{\prime}\right\rangle$ and $r_{2} \rightleftarrows *\left\langle w_{12} s^{\prime}, w_{22} s^{\prime}\right\rangle$. We take $u_{1} \rightleftarrows^{*}\left(\left\langle w_{11}, w_{21}\right\rangle\right) t^{\prime}$ and $r_{2} \rightleftarrows^{*}\left(\left\langle w_{12}, w_{22}\right\rangle\right) t^{\prime}$.

(b) $a_{2} s^{\prime} \rightleftarrows^{*}\left\langle w_{1} s^{\prime}, w_{2} s^{\prime}\right\rangle, r_{1} \rightleftarrows^{*}\left\langle a_{1} s^{\prime}, w_{2} s^{\prime}\right\rangle$ and $r_{2} \rightleftarrows^{*} w_{2} s^{\prime}$. So we take $u_{1}=\left(\left\langle a_{1}, a_{2}\right\rangle\right) t^{\prime}$ and $u_{2}=w_{2} t^{\prime}$, the symmetric cases are analogous.

(c) $r_{1} \rightleftarrows^{*} a_{1} s^{\prime}$ and $r_{2} \rightleftarrows^{*} a_{2} s^{\prime}$, in which case we take $u_{1}=a_{1} t^{\prime}$ and $u_{2}=a_{2} t^{\prime}$ the symmetric case is analogous.

- $s=\Lambda X . a, t=\Lambda X . t^{\prime}$, and $a \hookrightarrow t^{\prime}$, with $a \rightleftarrows^{*}\left\langle a_{1}, a_{2}\right\rangle$ and $s \rightleftarrows^{*} \Lambda X .\left\langle a_{1}, a_{2}\right\rangle X . a_{2}$. Therefore, by the induction hypothesis, there exists $u_{1}^{\prime}, u_{2}^{\prime}$ such that either $\left(a_{1} \rightarrow u_{1}^{\prime}\right.$ and $\left.a_{2} \rightarrow u_{2}^{\prime}\right)$, or $\left(a_{1} \rightleftarrows^{*} u_{1}^{\prime}\right.$ and $\left.a_{2} \rightarrow u_{2}^{\prime}\right)$, or $\left(a_{1} \rightarrow u_{1}^{\prime}\right.$ and $\left.a_{2} \rightleftarrows^{*} u_{2}^{\prime}\right)$. Therefore, we take $u_{1}=\Lambda X . u_{1}^{\prime}$ and $u_{2}=\Lambda X . u_{2}^{\prime}$.

- $s=a[A], t=t^{\prime}[A]$, and $a \hookrightarrow t^{\prime}$, with $a \rightleftarrows^{*}\left\langle a_{1}, a_{2}\right\rangle$ and $s \rightleftarrows^{*}\left\langle a_{1}[A], a_{2}[A]\right\rangle$. Therefore, by the induction hypothesis, there exists $u_{1}^{\prime}, u_{2}^{\prime}$ such that either $\left(a_{1} \rightarrow u_{1}^{\prime}\right.$ and $\left.a_{2} \rightarrow u_{2}^{\prime}\right)$, or $\left(a_{1} \rightleftarrows * u_{1}^{\prime}\right.$ and $\left.a_{2} \rightarrow u_{2}^{\prime}\right)$, or $\left(a_{1} \rightarrow u_{1}^{\prime}\right.$ and $\left.a_{2} \rightleftarrows^{*} u_{2}^{\prime}\right)$. Therefore, we take $u_{1}=u_{1}^{\prime}[A]$ and $u_{2}=u_{2}^{\prime}[A]$.

\section{Detailed proofs of Section 5.5}

Lemma 5.32 (Adequacy of product). For all $r, s, A, B$ such that $r \in \llbracket A \rrbracket$ and $s \in \llbracket B \rrbracket$, we have $\langle r, s\rangle \in \llbracket A \wedge B \rrbracket$.

Proof. We need to prove that $K_{A \wedge B}^{X}\langle\langle r, s\rangle) \in \mathrm{SN}$. We proceed by induction on the number of projections in $K_{A \wedge B}^{X}$. Since the hole of $K_{A \wedge B}^{X}$ has type $A \wedge B$, and $K_{A \wedge B}^{X}(t)$ has type $X$ for any $t$ of type $A \wedge B$, we can assume, without lost of generality, that the context $K_{A \wedge B}^{X}$ has the form $K_{C}^{\prime X}\left(\pi_{C}\left((0)^{A \wedge B} \alpha_{1} \ldots \alpha_{n}\right)\right)$, where each $\alpha_{i}$ is either a term or a type argument. We prove that all $K_{C}^{\prime X}\left(\pi_{C}\left(\left\langle r \alpha_{1} \ldots \alpha_{n}, s \alpha_{1} \ldots \alpha_{n}\right\rangle\right)\right) \in$ SN by showing, more generally, that if $r^{\prime}$ and $s^{\prime}$ are two reducts of $r \alpha_{1} \ldots \alpha_{n}$ and $s \alpha_{1} \ldots \alpha_{n}$, then $K_{C}^{\prime X}\left(1 \pi_{C}\left(\left\langle r^{\prime}, s^{\prime}\right\rangle\right) D \in \mathrm{SN}\right.$. For this, we show that all its one step reducts are in SN, by induction on $\left|K_{C}^{\prime X}\right|+\left|r^{\prime}\right|+\left|s^{\prime}\right|$.

- If the reduction takes place in one of the terms in $\mathcal{T}\left(K_{C}^{\prime X}\right)$, in $r^{\prime}$, or in $s^{\prime}$, we apply the induction hypothesis.

- Otherwise, the reduction is a $(\pi)$ reduction of $\pi_{C}\left(\left\langle r^{\prime}, s^{\prime}\right\rangle\right)$, that is, $\left\langle r^{\prime}, s^{\prime}\right\rangle \rightleftarrows^{*}\langle v, w\rangle$, the reduct is $v$, and we need to prove $K_{C}^{\prime X}(v) \in \mathrm{SN}$. By Lemma 5.18, we have either:

$-v \rightleftarrows^{*}\left\langle r_{1}, s_{1}\right\rangle$, with $r^{\prime} \rightleftarrows^{*}\left\langle r_{1}, r_{2}\right\rangle$ and $s^{\prime} \rightleftarrows^{*}\left\langle s_{1}, s_{2}\right\rangle$. In such a case, by Lemma $5.29, v$ is the product of two reducible terms, so since there is one projection less than in $K_{A \wedge B}^{X}$, the first induction hypothesis applies.

$-v \rightleftarrows^{*}\left\langle r^{\prime}, s_{1}\right\rangle$, with $s^{\prime} \rightleftarrows^{*}\left\langle s_{1}, s_{2}\right\rangle$. In such a case, by Lemma $5.29, v$ is the product of two reducible terms, so since there is one projection less than in $K_{A \wedge B}^{X}$, the first induction hypothesis applies.

$-v \rightleftarrows^{*}\left\langle r_{1}, s^{\prime}\right\rangle$, with $r^{\prime} \rightleftarrows^{*}\left\langle r_{1}, r_{2}\right\rangle$. In such a case, by Lemma 5.29, $v$ is the product of two reducible terms, so since there is one projection less than in $K_{A \wedge B}^{X}$, the first induction hypothesis applies.

- $v \rightleftarrows^{*} r^{\prime}$, in which case, $C \equiv A$, and since $r \in \llbracket A \rrbracket$, we have $K_{A}^{\prime X}\left(r^{\prime}\right) \rightleftarrows * K_{A}^{\prime X}(v) \in \mathrm{SN}$.

$-v \rightleftarrows^{*} r_{1}$ with $r^{\prime} \rightleftarrows^{*}\left\langle r_{1}, r_{2}\right\rangle$, in which case, since $r \in \llbracket A \rrbracket$, we have $K_{C}^{\prime X}\left(\pi_{C}\left(r^{\prime}\right) \downarrow \in \mathrm{SN}\right.$ and $K_{C}^{\prime X}\left(\pi_{C}\left(r^{\prime}\right)\right) \rightarrow K_{C}^{\prime X}(v)$ hence $K_{C}^{\prime X}(v) \in \mathrm{SN}$.

- $v \rightleftarrows^{*} s^{\prime}$, in which case, $C \equiv B$, and since $s \in \llbracket B \rrbracket$, we have $K_{B}^{\prime X}\left(s^{\prime}\right) \rightleftarrows^{*} K_{B}^{\prime X}(v) \in \mathrm{SN}$.

$-v \rightleftarrows^{*} s_{1}$ with $s^{\prime} \rightleftarrows^{*}\left\langle s_{1}, s_{2}\right\rangle$, in which case, since $s \in \llbracket B \rrbracket$, we have $K_{C}^{\prime X}\left(\pi_{C}\left(s^{\prime}\right) \rrbracket \in \mathrm{SN}\right.$ and $K_{C}^{\prime X}\left(\pi_{C}\left(s^{\prime}\right)\right) \rightarrow K_{C}^{\prime X}(v)$ hence $K_{C}^{\prime X}(v) \in \mathrm{SN}$. 
Lemma 5.34 (Adequacy of type abstraction). For all $r, X, A, B$ such that $[X:=B] r \in \llbracket[X:=B] A \rrbracket$, we have $\Lambda X . r \in \llbracket \forall X . A \rrbracket$.

Proof. We proceed by induction on $M(r)$.

- If $r \rightleftarrows^{*}\left\langle r_{1}, r_{2}\right\rangle$, then by Lemma 4.12, then $A \equiv A_{1} \wedge A_{2}$ with $r_{1}$ of type $A_{1}$ and $r_{2}$ of type $A_{2}$, and so by Lemma 4.13, $[X:=B] r_{1}$ has type $[X:=B] A_{1}$ and $[X:=B] r_{2}$ has type $[X:=B] A_{2}$. Since $[X:=B] r \in \llbracket[X:=B] A \rrbracket$, we have $\left\langle[X:=B] r_{1},[X:=B] r_{2}\right\rangle \in \llbracket[X:=B] A \rrbracket$. By Lemma 5.29, $[X:=B] r_{1} \in \llbracket[X:=B] A_{1} \rrbracket$ and $[X:=B] r_{2} \in \llbracket[X:=B] A_{2} \rrbracket$. By the induction hypothesis, $\Lambda X . r_{1} \in \llbracket \forall X . A_{1} \rrbracket$ and $\Lambda X . r_{2} \in \llbracket \forall X . A_{2} \rrbracket$, then by Lemma 5.32, $\Lambda X . r \rightleftarrows^{*}\left\langle\Lambda X . r_{1}, \Lambda X . r_{2}\right\rangle \in$ $\llbracket\left(\forall X . A_{1}\right) \wedge\left(\forall X . A_{2}\right) \rrbracket$, and by Lemma 5.26, $\left(\forall X . A_{1}\right) \wedge\left(\forall X . A_{2}\right) \rrbracket=\llbracket \forall X . A \rrbracket$.

- If $r \not \not^{*}\left\langle r_{1}, r_{2}\right\rangle$, we need to prove that for any elimination context $K_{\forall X . A}^{X}$, we have $K_{\forall X . A}^{X}(\Lambda X . r\rangle \in$ SN.

Since $r$ and all the terms in $\mathcal{T}\left(K_{\forall X . A}^{X}\right)$ are in SN, we proceed by induction on $\left|K_{\forall X . A}^{X}\right|+|r|$ to show that all the one step reducts of $K_{\forall X . A}^{X}(\Lambda X . r)$ are in SN. Since $r$ is not a product, its only one step reducts are the following.

- If the reduction takes place in one of the terms in $\mathcal{T}\left(K_{\forall X . A}^{X}\right)$ or $r$, we apply the induction hypothesis.

- If $K_{\forall X . A}^{X}(\Lambda X . r)=K_{A}^{\prime X}((\Lambda X . r)[B])$ and it reduces to $\left.K_{A}^{\prime X} 0[X:=B] r \downarrow\right)$, as $[X:=B] r \in \llbracket A \rrbracket$, we have $K_{A}^{\prime X}([X:=B] r) \in \mathrm{SN}$.

- If $r \rightleftarrows^{*} \lambda x^{C} \cdot r^{\prime}$, then by Lemma 4.12, we have $A \equiv C \Rightarrow A^{\prime}$ with $r^{\prime}$ of type $A^{\prime}$ and $X \notin$ $F V(C)$, and so by Lemma $4.13,[X:=B] r^{\prime}$ has type $[X:=B] A^{\prime}$. By Lemma 5.28, we have $x \in \llbracket[X:=B] C \rrbracket$, and since $[X:=B] \lambda x^{C} \cdot r^{\prime} \in \llbracket[X:=B]\left(C \Rightarrow A^{\prime}\right) \rrbracket$, by Lemma 5.30, we have $[X:=B] r^{\prime} \in \llbracket[X:=B] A^{\prime} \rrbracket$. By the induction hypothesis, we have $\Lambda X . r^{\prime} \in \llbracket \forall X . A^{\prime} \rrbracket$, then by Lemma $5.33, \Lambda X . r \rightleftarrows^{*} \Lambda X . \lambda x^{C} . r^{\prime} \in \llbracket C \Rightarrow \forall X . A^{\prime} \rrbracket$, and by Lemma 5.26, since $X \notin F V(C)$, we have $\llbracket C \Rightarrow \forall X . A^{\prime} \rrbracket=\llbracket \forall X . A \rrbracket$. 